\title{
Walrasian Equilibrium in an Exchange Economy with Indivisibilities*
}

\author{
Jinpeng Ma \\ Department of Economics \\ Rutgers University-Camden, NJ 08102 \\ jinpeng@crab.rutgers.edu \\ Fusheng Nie \\ Philadephia Stock Exchange \\ FA, Philadelphia, PA19103 \\ niefr@phlx.com \\ Prelimilary Draft December 1,2001 \\ This Version April 19,2002
}

\begin{abstract}
This paper studies an exchange economy with indivisibilities. Our main goal is to see if a price system can function well in an economy (e.g., an economy with complementary preferences) that does not have a Walrasian equilibrium. We study the price adjustment processes governed by the Euler iterative scheme. We show that in an economy that has a Walrasian equilibrium, our price adjustment processes have a common uniform limit that is unique and converges to a Walrasian equilibrium price vector in finite time. Surprisingly, in an economy that does not have a Walrasian equilibrium, our price adjustment processes also have a common uniform limit that is unique and converges to a market equilibrium price vector in finite time. Moreover, market equilibrium prices coincide with Walrasian equilibrium ones in an economy that has a Walrasian equilibrium. Further, there are no prices other than the Walrasian or market equilibrium ones that have such a property of global stability.
\end{abstract}

\section{Introduction}

An equilibrium in an exchange economy is defined by the condition under which no goods are in excess demand or supply. If a market fails to clear, prices are expected to adjust to equilibrium such that the market clearing condition is eventually met. A question of interest is how a market or a price system operates if a market fails to have an equilibrium. In such a market some goods are always in excess demand or supply no matter what are the prices. So what may be the market outcome?

Now consider an economy where an equilibrium exists. One expects that the market outcome is at some equilibrium. But such a claim is based on the belief that a price system will soon converge

\footnotetext{
${ }^{*}$ The paper was initially prepared for the NATO Advance Research Workshop on Mathematical Theories of Allocation of Discrete Resources cosponsored by Sabanci University in Istanbul in December 2001. We thank the participants of the workshop for helpful comments and suggestions.
} 
to the original equilibrium or migrate to a new equilibrium once it deviates from the equilibrium. Thus, the property of global or local stability of economic equilibria is very important in both theory and applications. Fisher (1983) pointed out in a general equilibrium framework that "[t]he proposition that the equilibria of economic models are not only stable but that convergence to a neighborhood of equilibrium is achieved relatively quickly turns out to be a necessary foundation for the equilibrium analysis of economic theory."

At equilibrium, the excess demand function, defined on the price space, may happen to be a set-valued map. This means that it may contain elements under which a market fails to clear. At equilibrium, what may be the reason that agents in the marketplace will definitely select the desired one from the excess demand function such that a market clears? If there is a chance that some other alternatives may be chosen where a market fails to clear, then can we still believe in an equilibrium to be the outcome of a market?

This paper studies these and the related issues in an exchange economy with multiple indivisible goods studied in Bikhchandani and Mamer (1997) and Gül and Stacchetti (1999). ${ }^{1}$ Bikhchandani and Mamer (1997) used the linear programming to provide a necessary and sufficient condition for the existence of a Walrasian equilibrium. Gül and Stacchetti (1999) provided two sufficient conditions, the no complementarities and the single improvement property on agents' utility functions, and showed that their two conditions are equivalent to the gross substitutes condition in Kelso and Crawford (1982). They then invoked the nonempty core theorem in Kelso and Crawford (1982) to show that a Walrasian equilibrim exists under their two new conditions.

Despite the generality of the gross substitutes condition, there are still many economies that do not satisfy it and have no Walrasian equilibrium. In particular, an economy may not have a Walrasian equilibrium when agents' utility functions have complementary property. Milgrom (2000) provided a general result that shows that an economy may not have a Walrasian equilibrium if even one agent has preferences that are complementary, and all the rest agents have preferences that satisfy the mutual (gross) substitutes condition. Thus, what may be the market outcome in such an economy with complementary utility functions? An answer to the question has an important implication to the design of an auction mechanism for the sale of multiple-unit objects.

In Section 5 we discuss the existence of a Walrasian equilibrium and its relationship to the lottery and sunspot equilibrium. Here we are concerned with the issue if lotteries and sunspots can provide new equilibrium to study the kind of markets that do not have a Walrasian equilibrium. Prescott and Townsend (1984) introduced lotteries into a variety of economies with private information and a continuum of consumers and showed that with the help of lotteries new equilibria can emerge. In contrast, Garratt (1995) studied an economy with a finite number of consumers and indivisible goods and showed that any lottery equilibrium is either a Walrasian equilibrium or a sunspot equilibrium. But there are Walrasian and sunspot equilibria that are not lottery equilibria. Garrat et al (2001) studied an economy with a finite number of consumers and a continuous sunspot variable and showed that the two notions of lottery and sunspot equilibrium are equivalent in the equilibrium allocations. Garratt, Keister and Shell (2001) studied an economy with a finite number of consumers and a finite sunspot variable and showed that a substantial difference exists between lottery and sunspot equilibrium. Some new instrument has to be brought into their model to bridge the gap of the two notions. The economies studied in these papers are along the Arrow-Debreu general equilibrium framework that is different from ours. But the question raised in these papers is equally important to us. In our economy, we show that a Walrasian equilibrium exists iff a lottery

\footnotetext{
${ }^{1}$ This exchange economy is closely related to the job matching model in Kelso and Crawford (1982). But it should be aware that the utility function for a worker in Kelso and Crawford (1982) is more sophisticated and depends on not only the salary offered by a firm but also the name of that firm. This is quite different from the exchange economy studied here where agents are not concerned with the name of the owner of a commodity.
} 
equilibrium exists. We also introduce a finite sunspot variable into our economy and show that a Walrasian equilibrium exists iff a sunspot equilibrium exists. So lotteries and sunspots are not helpful to provide a new equilibrium.

Our paper is largely motivated by those price adjustment processes in discrete time in Kelso and Crawford (1982), Demange, Gale and Sotomayor (1986), Gül and Stacchetti (2000), and Milgrom (2000). Kelso and Crawford (1982) studied a job matching market and provided an ascending salary-adjustment process in discrete time that converges to an efficient core outcome (i.e. a Walrasian equilibrium) in finite time. Demange, Gale and Sotomayor (1986) studied the assignment problem (Shapley and Shubik (1972)) and used the Hall theorem to design an ascending price adjustment process in discrete time that converges to the minimum Walrasian equilibrium prices in finite time. Gül and Stacchetti (2000) studied the economy in Bikhchandani and Mamer (1997) and Gül and Stacchetti (1999) and provided an ascending price adjustment process in discrete time that converges to the minimum Walrasian equilibrium prices in finite time. The two convergence results in Kelso and Crawford (1982) and Gül and Stacchetti (2000) depend on the gross substitutes condition. Under the mutual (gross) substitutes condition, Milgrom (2000) showed that the price adjustment process generated by the new simultaneous ascending price auction used for the sale of spectrum licenses in the U.S. converges to a Walrasian equilibrium within finite time. Ma (1998b) studied the exchange economy in Bikhchandani and Mamer (1997) and Gül and Stacchetti (1999) and showed that every ascending price process in discrete time that has the excess demand property converges to a Walrasian equilibrium in finite time. This applies to an exchange economy that has a Walrasian equilibrium but may not satisfy the gross substitutes condition. These price adjustment processes share some common features. First, the convergence of each price adjustment process above does not have the global or local stability because each convergence result only holds when it starts with one particular price vector (most of the time the zero vector). It is unclear if the convergence results still hold if the processes start with a price vector, say, in a neighborhood of an equilibrium. Second, prices are increased in each round for a specific set of objects that are in excess demand. This requires the auctioneer to have an aggregate coordination on agents' behavior and instruct each agent which bundle to choose. In particular, when an agent's demand set in a round contains multiple bundles, a tie-breaking device, which is often thought to be natural under such a situation, is not allowed. Third, these processes depend on the existence of a Walrasian equilibrium and do not provide a guidance for the issue what may be the market outcome in an economy that does not have a Walrasian equilibrium. When a Walrasian (competitive) equilibrium does not exist, "[t]here is an inherent limitation in the very conception of the auction as a process for discovering a competitive allocation and competitive prices in that case" (Milgrom (2000)). These limitations motivate our study in this paper for new price adjustment processes.

Our approach is somehow different and may be illustrated by an example borrowed from Milgrom (2000). Consider an exchange economy with two agents $j$ and $k$, and two objects 1 and 2 . The two objects are initially owned by a seller whose reservation prices for each individual object and the bundle are zero. Agents' utility functions are as follows:

$$
\begin{gathered}
u_{j}(\{1\})=1, \quad u_{j}(\{2\})=1, u_{j}(\{1,2\})=3 \\
u_{k}(\{1\})=1 \frac{3}{4}, \quad u_{k}(\{2\})=1 \frac{3}{4}, u_{k}(\{1,2\})=2 \frac{3}{4} .
\end{gathered}
$$

The utility function for agent $j$ is complementary. There is no Walrasian equilibrium in this economy. Now suppose that a price adjustment process follows such a simple principle: At each round, if an object is in excess demand (supply), its price is raised (lowered) by a positive $\delta .{ }^{2}$

\footnotetext{
${ }^{2}$ If at a round, the adjusted price is negative, then set it to be zero.
} 
Since an agent's demand function is a set-valued map, such a process generates a family of Euler polygonal arcs, starting from an arbitrary nonnegative price vector. Since this economy does not have a Walrasian equilibrium, there always exists some object that is in excess demand or supply. So one may expect that none of the price adjustment process in the family really converges. On the contrary, we will show that given any $\epsilon>0$, a positive $\delta$ can be found such that each price process or Euler polygonal arc in this family converges in finite time to the price vector $(1.5,1.5)$ with $\epsilon$-accuracy. Moreover, as $\delta$ approaches zero, all Euler polygonal arcs in the family have a uniform limit that is unique. In addition, we will argue why the two objects should be allocated to agent $j$ at the prices $(1.5,1.5)$ by designing an implementation game. So the seller extracts all surplus in this economy. Complementary preferences benefit the seller. ${ }^{3}$

The main results of this paper are that the results for this example can be generalized to a more general exchange economy with indivisible goods. Thus, we obtain some insights that cannot be obtained from the above price adjustment processes in the literature. For example, in an economy without a Walrasian equilibrium, one may expect that there is no price vector that can be stable for any price adjustment process. Our results will show that this is incorrect.

Precisely, let $[0, b]$ be the time interval, where $b$ is some large finite number. Let

$$
\Gamma=\left\{t_{0}, t_{1}, \cdots, t_{j}, t_{j+1}, \cdots, t_{\mu-1}, t_{\mu}\right\}
$$

be a uniform partition of $[0, b]$, where $t_{0}=0$ and $t_{\mu}=b$. We assume that our price adjustment process follows the Classic Euler iterative scheme:

$$
\frac{p\left(t_{j}\right)-p\left(t_{j-1}\right)}{t_{j}-t_{j-1}}-f\left(p\left(t_{j-1}\right)\right)=0, j=1,2, \cdots, \mu-1, \mu,
$$

where

$$
f\left(p\left(t_{j-1}\right)\right) \in \sum_{i} \operatorname{co}\left(D_{i}\left(p\left(t_{j-1}\right)\right)\right)-\left[\begin{array}{c}
1 \\
\vdots \\
1
\end{array}\right],
$$

and $c o\left(D_{i}(\cdot)\right)$ is the convex hull of bundles in $D_{i}(\cdot){ }^{4}$ Therefore, $f\left(p\left(t_{j-1}\right)\right)$ is an excess demand bundle at $t_{j-1}$, where agents are free to choose any bundle in their demand sets $D_{i}(\cdot)$ or use a tiebreaking device to choose a bundle, if needed. This price adjustment process follows the principle that the price of a commodity is adjusted higher if it is in excess demand and lower if it is in excess supply, much like what we have taught our students in the Principles of Economics. ${ }^{5}$ Given a partition $\Gamma$ and an initial prices $p_{0} \in R_{+}^{n}$, a sequence of prices, $p\left(t_{0}\right)=p_{0}, p\left(t_{1}\right), \cdots, p\left(t_{j}\right), \cdots, p\left(t_{\mu}\right)$, is generated by $E$ for a given selection $f$. For a given partition $\Gamma$ and an initial prices $p_{0}$, there are many such sequences since each $D_{i}(\cdot)$ often contains multiple bundles. We are interested in the uniform limit of such a family of sequences when the number of partitions $\mu$ approaches $\infty$ (i.e. in continuous time or in an environment with perfectly flexible prices).

Our main results are as follows:

\footnotetext{
${ }^{3}$ In the markets of computers, furniture, and cars etc, sellers of course prefer to have buyers whose preferences are complement rather substitute. It is quite intuitive why the seller gets better off in this example.

${ }^{4}$ Our results still hold without taking the convex hull.

${ }^{5}$ The economic principles about equilibria also bear fruits in the Golf world. Tiger Woods wrote on page 12 in his year2001 book "How I Play Golf", published by Warner Books, that "[s]uccess in Golf is finding equilibrium, accepting the fact that it is a game of ups and downs and learning something everytime you tee it up. Finding the balance is a matter of trial and error."
} 
- The uniform limit is unique that is the only absolutely continuous function $p(t)$ defined on $[0, b]$ that, together with its derivative with respect to $t$, satisfies the differential inclusion

$$
\frac{d p(t)}{d t} \in \sum_{i} c o\left(D_{i}(p(t))\right)-\left[\begin{array}{c}
1 \\
\vdots \\
1
\end{array}\right], p(0)=p_{0} .
$$

- Let $p(t)$ be the uniform limit defined on $[0, b]$. Then, in an economy that has a Walrasian equilibrium, there exists a finite time $t^{*}$ such that $p(t)$ is a Walrasian price vector for all $t \geq t^{*}$.

- Let $p(t)$ be the uniform limit defined on $[0, b]$. Then, in an economy that does not have a Walrasian equilibrium, there exist a nonempty convex compact subset $\Theta$ of $R_{+}^{n}$ and a finite time $t^{*}$ such that $p(t)$ is in $\Theta$ for all $t \geq t^{*}$.

A price vector $p$ in $\Theta$ is defined as a market equilibrium price vector. It is not Walrasian because the market clearing condition may not satisfy. If an economy has a Walrasian equilibrium, then $\Theta$ is exactly the set of all Walrasian price vectors. Moreover, there are no other prices than Walrasian or market equilibrium prices that have such a property of global stability under the dynamics of (E). These results are shown in Section 6. They depend largely on the mathematical tool developed in the nonsmooth analysis and the related control theory. In particular, the Clarke regular functions and their sub-differentials are important to us. We introduce them in Section 3. Among many others, the Upper Envelope Theorem (Lemma 6) plays an important role in our analysis throughout the paper. It connects the convex hulls of our demand functions $D_{i}(\cdot)$ with the sub-differentials $\partial v_{i}$ of the surplus functions $v_{i}(\cdot)$. This result is an application of Theorem 5 in Aubin and Cellina (1984, pp.36) and can be derived from the Chain rule in Clarke et al (1988).

The rest of the paper is organized as follows. Section 2 introduces the model. Section 4 introduces a number of preliminary results and the directinal derivatives of the surplus functions $v_{i}(\cdot)$. Section 7 discusses truncated strategies and presents an implementation game in the allocation of objects that can be used for the design of an auction mechanism.

\section{The Model}

Consider an exchange economy with a finite number of indivisible objects indexed by $k, k \in N=$ $\{1,2, \cdots, n\}$, and a finite number of agents indexed by $i, i \in M=\{1,2, \cdots, m\}$. Each agent $i$ 's utility function $u_{i}: 2^{N} \rightarrow R_{+}$is defined by a set function $u_{i}(S)$ over all object bundles $S \subset N$ such that $u_{i}(\emptyset)=0$ and $u_{i}(S) \leq u_{i}(T)$ for any two object bundles $S, T \subset N$ such that $S \subset T$. Let $\mathcal{E}$ denote this exchange economy.

A feasible allocation $X$ in th economy $\mathcal{E}$ is a partition $\left(X_{0}, X_{1}, \cdots, X_{m}\right)$ of the set of objects $N$, in which agent $i, i \in M$, is allocated the bundle $X_{i} . X_{0}$ is the unallocated bundle.

Given a price vector $p \in R_{+}^{n}$, agent $i$ 's trading profit function $v_{i}: 2^{N} \times R_{+}^{n} \rightarrow R$ is defined by

$$
v_{i}(S, p)=u_{i}(S)-\sum_{a \in S} p_{a}
$$

and his demand correspondence $D_{i}: R^{n} \rightarrow 2^{N}$ is defined by

$$
D_{i}(p)=\left\{S \subset N: v_{i}(S, p) \geq v_{i}(T, p), \forall T \subset N\right\}
$$


Define $v_{i}(p)=v_{i}(S, p)$ for $S \in D_{i}(p)$. The function $v_{i}(\cdot)$ is the consumer surplus function for agent $i$. A pair $(X, p)$ of a feasible allocation $X$ and a price vector $p$ is a Walrasian (competitive) equilibrium if $p_{a}=0$ for all $a \in X_{0}$ and $X_{i} \in D_{i}(p)$ for all agents $i$. This definition of a Walrasian equilibrium is standard in the literature.

This economy is closely related to the job matching market in Kelso and Crawford (1982) and has been recently studied in Bikhchandani and Mamer (1997) and Gül and Stacchetti (1999) for the existence issue. It is similar to the model used in Milgrom (2000) to study the spectrum license auction markets. Ma (1998a) studied a related model in which agents are initially endowed with the objects and provided a necessary and sufficient condition for the existence of a Walrasian equilibrium by generating a coalitional form game. There are also extensive studies of economies with indivisible goods in the Arrow-Debreu general equilibrium framework with the existence issue, see, e.g., Danilov and Koshevoy (2001), Laan, Talman and Yang (2001), and references therein.

\section{Sub-differentials}

In this section we introduce some definitions and results in the nonsmooth analysis from Clarke et al (1988). In particular, the class of the Clarke regular functions plays an important role in our analysis. ${ }^{6}$

Let $\varphi: Y \rightarrow R \cup\{+\infty\}$ be an extended real-valued mapping on $Y$ where $Y \subset R_{+}^{n}$. Define the effective domain of $\varphi$ by

$$
\operatorname{Dom}(\varphi)=\{y \in Y: \varphi(y)<\infty\} .
$$

The function $\varphi$ is proper if $\operatorname{Dom}(\varphi)$ is nonempty. It is (locally) Lipschitz near $y$ if for some constant $K$, for all points $y, y^{\prime}$ in some neighborhood of $y$, we have

$$
\left|\varphi(y)-\varphi\left(y^{\prime}\right)\right| \leq K d\left(y, y^{\prime}\right)
$$

The smallest such $K$ for which the above inequality holds for all points in a given set $Y$ is called the Lipschitz rank of $\varphi$ relative to the set $Y$. Under such a situation, we say that the function $\varphi$ is globally Lipschitz on the set $Y$. A locally Lipschitz function on $Y$ is continous on $Y$. A globally Lipschitz function $\varphi$ on $Y$ is uniformly continuous on $Y$.

The directional direvative of $\varphi$ at $y \in Y$ in the direction $w \in Y$ is defined as

$$
\varphi^{\prime}(y ; w)=\lim _{t \downarrow 0} \frac{\varphi(y+t w)-\varphi(y)}{t}
$$

when the limit exists. It is Gâteaux differentiable at $y$ if the limit in (1) exists for all $w \in Y$, and there exists an element $\varphi^{\prime}(y) \in Y$ (called the Gâteaux derivative) that satisfies

$$
\varphi^{\prime}(y ; w)=<\varphi^{\prime}(y), w>, \forall w \in Y .
$$

A vector $\eta \in R^{n}$ is a sub-gradient of $\varphi$ at $y \in \operatorname{Dom}(\varphi)$ if for all directions $w \in Y$ the following holds

$$
<\eta, w>\leq \varphi^{\prime}(y ; w)
$$

The sub-differentiable of $\varphi$ at $y$, denoted by $\partial \varphi(y)$, is the set of all sub-gradients of $\varphi$ at $y$ (possibly empty), i.e.,

$$
\partial \varphi(y)=\left\{\eta \in R^{n}:<\eta, w>\leq \varphi^{\prime}(y ; w), \forall w \in Y\right\} .
$$

\footnotetext{
${ }^{6}$ See Royden (1988) for some other conceptions and results used below.
} 
The Clarke directional direvative $\varphi^{0}(y ; w)$ of $\varphi$ at $y$ in the direction $w$ is defined as follows

$$
\varphi^{0}(y ; w)=\limsup _{\substack{x \rightarrow y \\ t \downarrow 0}} \frac{\varphi(x+t w)-f(x)}{t} .
$$

A function $\varphi$ is regular at $y$ if it is Lipschitz near $y$ and for all $w$ the directional derivative $\varphi^{\prime}(y ; w)$ exists and $\varphi^{\prime}(y ; w)=\varphi^{0}(y ; w)$. It is regular on $Y$ if it is regular for all $y \in Y$. Every convex function on a compact set $Y$ is regular Lipschitzian. Convex functions that are Lipschitz near $y$ are regular at $y$.

For a regular function $\varphi$, we know that

$$
\partial \varphi(y)=\left\{\eta \in R^{n}:<\eta, w>\leq \varphi^{0}(y ; w), \forall w \in Y\right\} .
$$

If the function $\varphi$ is convex and locally Lipschitzian at $y$, we know that $\partial \varphi(y)$ is a nonempty, compact, and convex set. Moreover, it is upper semicontinuous at $y$ in the sense that for all $\epsilon>0$ there exists $\delta>0$ such that

$$
\|x-y\|<\delta \Longrightarrow \partial \varphi(x) \subset \partial \varphi(y)+\epsilon B
$$

where $B$ is the open unit ball in $Y$. The sub-differential $\partial \varphi$ can be expressed as

$$
\partial \varphi(y)=\left\{\eta \in R^{n}: \varphi(w)-\varphi(y) \geq<\eta, w-y>, \forall w \in Y\right\}
$$

also see Rockafellar (1970). Moreover, it is known that $\partial \varphi$ is a maximal monotone operator, i.e., $<f_{1}-f_{2}, y_{1}-y_{2}>\geq 0$ if $f_{j} \in \partial \varphi\left(y_{j}\right), j=1,2$, and there is no other monotone set-valued map whose graph contains strictly the graph of $\partial \varphi$ (Aubin and Cellina (1984)).

If the function $\varphi$ is Gâteaux differentiable at $y$, then we know that $\partial \varphi(y)=\{\nabla \varphi(y)\}$. If $\varphi$ is continuous at $y$ and $\partial \varphi$ is a singleton, then $\varphi$ is Gâteaux differentiable at $y$. For a convex function $\varphi, y$ minimizes $\varphi$ on $Y$ iff $0 \in \partial \varphi(y)$.

For any two regular functions $\varphi$ and $\psi$ at $y$, the $\operatorname{sum} \varphi+\psi$ is regular at $y$ and

$$
\partial(\varphi+\psi)(y)=\partial \varphi(y)+\partial \psi(y)
$$

\section{Preliminaries}

Define $V: R_{+}^{n} \rightarrow R$ as

$$
V(p)=\sum_{a \in N} p_{a}+\sum_{i=1}^{m} v_{i}(p) .
$$

Smith $(1962,1965)$ defined an economic rent function for a single commodity market and used it to study the double auction markets. This function $V(\cdot)$ is an extension of the economic rent function in Smith $(1962,1965)$ for a single commodity market to our economy with multiple indivisible objects. Our next lemma shows that the functions $v_{i}(\cdot), i \in M$, and $V(\cdot)$ are all regular on $R_{+}^{n}$.

Lemma 1. The function $v_{i}$ is convex, uniformly continuous, and globally Lipschitz on $R_{+}^{n}$ with $\operatorname{rank} \sqrt{n}$.

Proof. Clearly $v_{i}(p)$ is a convex function in $p$. To complete the proof, we now show that $v_{i}(p)$ is Lipschitz with rank $\sqrt{n}$ on $R_{+}^{n}$. For any two price vectors $p^{\prime}$ and $p^{\prime \prime}$ in $R_{+}^{n}$, define $l:[0,1] \rightarrow R_{+}^{n}$ by

$$
l(t)=p^{\prime}+t\left(p^{\prime \prime}-p^{\prime}\right) .
$$


Then, there exist $t_{0}=0<t_{1}<\cdots<t_{k-1}<t_{k}=1$ such that on each line segment $l\left[t_{j}, t_{j+1}\right]=$ $\left\{p^{\prime}+t\left(p^{\prime \prime}-p^{\prime}\right): t \in\left[t_{j}, t_{j+1}\right]\right), v_{i}(p)$ is determined by some $v_{i}\left(S_{j}, p\right)$. That is,

$$
v_{i}(p)=v_{i}\left(S_{j}, p\right) \text { for all } p \in l\left[t_{j}, t_{j+1}\right], j=0,1, \cdots, k-1 \text {. }
$$

Now, we have

$$
\left|v_{i}\left(p^{\prime \prime}\right)-v_{i}\left(p^{\prime}\right)\right| \leq \sum_{j=0}^{j=k-1}\left|v_{i}\left(p^{j+1}\right)-v_{i}\left(p^{j}\right)\right|
$$

where $p^{j}=p^{\prime}+t_{j}\left(p^{\prime \prime}-p^{\prime}\right)$. Each summand on the right side of (2) is controlled by

$$
\begin{aligned}
\left|v_{i}\left(p^{j+1}\right)-v_{i}\left(p^{j}\right)\right| & =\left|\left(u_{i}\left(S_{j}\right)-\sum_{a \in S_{j}} p_{a}^{j+1}\right)-\left(u_{i}\left(S_{j}\right)-\sum_{a \in S_{j}} p_{a}^{j}\right)\right| \\
& =\left|\sum_{a \in S_{j}}\left(p_{a}^{j+1}-p_{a}^{j}\right)\right| \\
& \leq \sum_{a \in S_{j}}\left|p_{a}^{j+1}-p_{a}^{j}\right| \\
& \leq \sum_{a \in N}\left|p_{a}^{j+1}-p_{a}^{j}\right| \\
& \leq \sqrt{n} d\left(p^{j}, p^{j+1}\right),
\end{aligned}
$$

where $d$ is the Euclidean distance in $R_{+}^{n}$. Plug (3) into (2), we get

$$
\left|v_{i}\left(p^{\prime \prime}\right)-v_{i}\left(p^{\prime}\right)\right| \leq \sum_{j=0}^{k-1} \sqrt{n} d\left(p^{j}, p^{j+1}\right)=\sqrt{n} d\left(p^{\prime}, p^{\prime \prime}\right) .
$$

This completes the proof.

Let $V=\max _{X} \sum_{i \in M} u_{i}\left(X_{i}\right)$ be the maximum total social welfare in the economy $\mathcal{E}$, where the maximum is taken over all feasible allocations $X$. An allocation $X^{*}$ is optimal if $V=\sum_{i \in M} u_{i}\left(X_{i}^{*}\right)$. Next we show that a Walrasian equilibrium exists if and only if $V(p)=V$ for some $p$. This is essentially the first and the second welfare theorem. It is also related to the necessary and sufficient condition in Bikhchandani and Mamer (1997). This result first appeared in Ma (1998b).

Lemma 2. (a). For all price vectors $p \in R_{+}^{n}, V(p) \geq V$; (b). A price vector $p \in R_{+}^{n}$ is Walrasian if and only if $V(p)=V$.

Proof. For all optimal allocations $X^{*}, v_{i}(p) \geq u_{i}\left(X_{i}^{*}\right)-\sum_{a \in X_{i}^{*}} p_{a}$ for all $i \in M$. Therefore,

$$
V(p) \geq \sum_{i \in M}\left[u_{i}\left(X_{i}^{*}\right)-\sum_{a \in X_{i}^{*}} p_{a}\right]+\sum_{k \in N} p_{k} \geq V
$$

This proves (a).

Let $p$ be a price vector such that $V(p)=p$. We show that for all $i \in M$, and all optimal allocations $X^{*}, X_{i}^{*} \in D_{i}(p)$. Suppose on the contrary that this is not true. Then there exists some $X^{*}$ such that $X_{i}^{*} \notin D_{i}(p)$ for some $i \in M$ Then

$$
V=V(p)
$$




$$
\begin{aligned}
& =\sum_{i \in M} v_{i}(p)+\sum_{k \in N} p_{k} \\
& >\sum_{i \in M}\left[\left(u_{i}\left(X_{i}^{*}\right)-\sum_{a \in X_{i}^{*}} p_{a}\right]+\sum_{k \in N} p_{k}\right. \\
& \geq V,
\end{aligned}
$$

a contradiction. Therefore for all optimal allocations $X^{*}, X_{i}^{*} \in D_{i}(p)$ for all $i \in N$. Moreover, it follows from above that $p_{a}=0$ for all $a$ such that $a \notin \cup_{i} X_{i}^{*}$. This shows that $p$ is Walrasian.

To show the other side, let $(p ; X)$ be a Walrasian equilibrium. Then

$$
V \leq V(p)=V+\sum_{a \in X_{0}} p_{a}=V
$$

since $p_{a}=0$ for all $a \in X_{0}$. This completes the proof of the lemma.

Thus, for an economy without a Walrasian equilibrium, we always have that $V(p)>V$ for all $p \in R_{+}^{n}$. Moreover, if $\min _{p} V(p)>V$, then we can conclude that a Walrasian equilibrium does not exist.

Example 3. Consider the following economy with two agents $j$ and $i$. Agents $j$ and $i$ have utility functions as follows (Kelso and Crawford (1982)):

$$
\begin{array}{lll}
u_{j}(\{1\})=4, & u_{j}(\{2\})=4, \quad u_{j}(\{3\})=4+\epsilon_{1} \\
u_{j}(\{1,2\})=7+\epsilon, & u_{j}(\{1,3\})=7, \quad u_{j}(\{2,3\})=7 \\
u_{j}(\{1,2,3\})=9 & \\
u_{i}(\{1\})=4+\epsilon_{2}, & u_{i}(\{2\})=4, \quad u_{i}(\{3\})=4 \\
u_{i}(\{1,2\})=7, \quad u_{i}(\{1,3\})=7, & u_{i}(\{2,3\})=7+\epsilon \\
u_{i}(\{1,2,3\})=9 &
\end{array}
$$

where $\epsilon \in[0,1]$ and $\epsilon_{1}, \epsilon_{2} \in[0,3]$. Clearly, these utility functions are weakly monotone.

Kelso and Crawford (1982) showed that their job matching market with parameters $\epsilon_{1}=\epsilon_{2}=\frac{1}{4}$, and $\epsilon=\frac{1}{2}$ has an empty core (i.e. no Walrasian equilibrium) by means of the core conditions. We may use Lemma 2 to provide a proof of that. Observe that the function $V(p)$ reaches its minimum at the price vector $p^{*}=\left(2 \frac{3}{4}, 3 \frac{1}{4}, 2 \frac{3}{4}\right)$ with $V\left(p^{*}\right)=11 \frac{3}{4}$. But $V=11 \frac{1}{2}$. Thus, Lemma 2 shows that this economy with the given parameters does not have a Walrasian equilibrium. Indeed, at $p^{*}$, we have

$$
D_{j}\left(p^{*}\right)=\{\{3\},\{1,2\},\{1,3\}\}, \quad D_{i}\left(p^{*}\right)=\{\{1\},\{1,3\},\{2,3\}\} .
$$

Clearly, the market clearing condition is violated at $p^{*}$.

Example 4 (Roth and Sotomayor (1990,pp.179)). An economy with complementary preferences. Let $N=\{1,2\}$ and $M=\{j, k\}$. The preferences are as follows:

$$
\begin{aligned}
& u_{j}(\{1\})=4, u_{j}(\{2\})=1, u_{j}(\{1,2\})=10 \\
& u_{k}(\{1\})=8, u_{k}(\{2\})=5, u_{k}(\{1,2\})=9 .
\end{aligned}
$$


The surplus functions are as follows:

$$
\begin{aligned}
& v_{j}(p)= \begin{cases}1-p_{2} & \text { if } p_{1} \geq 9, p_{2} \leq 1 \\
4-p_{1} & \text { if } p_{1} \leq 4, p_{2} \geq 6 \\
10-p_{1}-p_{2} & \text { if } p_{1} \leq 9, p_{2} \leq 6, p_{1}+p_{2} \leq 10 \\
0 & \text { otherwise }\end{cases} \\
& v_{k}(p)= \begin{cases}9-p_{1}-p_{2} & \text { if } p_{1} \leq 4, p_{2} \leq 1 \\
8-p_{1} & \text { if } p_{1} \leq 8, p_{2} \geq 1, p_{1}-p_{2} \leq 3 \\
5-p_{2} & \text { if } p_{1} \geq 4, p_{2} \leq 5, p_{1}-p_{2} \geq 3 \\
0 & \text { otherwise }\end{cases}
\end{aligned}
$$

Then

$$
V(p)=v_{j}(p)+v_{k}(p)+p_{1}+p_{2} .
$$

Roth and Sotomayor (1990) showed that the core of this economy is empty. We may use Lemma 2 to provide a proof of this. Note that $V=10$ and $V(p)$ reaches its minimum at $p^{* *}=\left(6 \frac{1}{2}, 3 \frac{1}{2}\right)$, with $V\left(p^{* *}\right)=11 \frac{1}{2}$. Thus, there does not exist a Walrasian equilibrium in this economy. Indeed, we have that

$$
D_{j}\left(p^{* *}\right)=\{\emptyset,\{1,2\}\}, D_{k}\left(p^{* *}\right)=\{\{1\},\{2\}\} .
$$

Note that the "demand curve" of one object in the above two examples depends on the presence of the other object(s). This interdependence in the demand curves across objects is a general feature of our model that makes it hard to analyze.

To conclude this section, we would like to mention the implication of our main results when they apply to these two examples. Our results will show that the price adjustment process of (E) in its limit when the number of partitions approaches $\infty$ will converge in finite time to the price vectors $p^{*}$ in Example 3 and $p^{* *}$ in Example 4 and be stable at there, starting from any initial price vector $p_{0}$ in the corresponding domains $\operatorname{Dom}(V(\cdot))$. Moreover, there are no other price vectors in Examples 3 and 4 that have such a property of stability. Thus, our study of this paper contributes to the literature by answering the question what may be the outcomes in an economy without a Walrasian equilibrium. This is especially important because there are markets for the sale of spectrum licenses that may not have a Walrasian equilibrium; see Milgrom (2000) for examples and a detailed discussion.

\subsection{Upper Envelope Theorem}

Define $e: 2^{N} \rightarrow R_{+}^{n}$ by $e_{k}(S)=1$ if $k \in S$ and $e_{k}(S)=0$ otherwise. Then, $v_{i}(S, p)=u_{i}(S)-<$ $e(S), p>, v_{i}(p)=\max _{S \in 2^{N}} v_{i}(S, p)$, and $D_{i}(p)=\left\{S: v_{i}(S, p)=v_{i}(S)\right\}$. Let $c o\left(D_{i}(p)\right)=c o\{e(S)$ : $\left.S \in D_{i}(p)\right\}$, where $c o(Z)$ denotes the convex hull of a set $Z$. Let $S_{1}, S_{2}, \cdots, S_{2^{n}}$, where $S_{1}=\emptyset$, be an enumeration of all the subsets of $N$ and $\Sigma=\left\{S_{1}, S_{2}, \cdots, S_{2^{n}}\right\}$.

We now provide two versions of the Envelope Theorem. Milgrom and Segal (2000) have provided a detailed study of the Envelope Theorem when the choice sets are arbitrary, with parameters located in the interval $[0,1]$. Since our parameters are in $R_{+}^{n}$, we need the directional derivatives for the Envelope Theorem. Our main observation is however the Upper Envelope Theorem expressed 
in Lemma 6. This result depends on the quasi-linear structure of the surplus functions and their regularity properties.

The following is a sort of an Envelope Theorem expessed in the directional derivatives. A corollary of it is that all directional derivatives $v_{i}^{\prime}(p ; w)$ exist on $R_{+}^{n}$. The existence result of the directional derivatives of Proposition 4.3 in Clarke et al $(1988$, pp.80) depends on the facts that $v_{i}(p)$ is convex and Lipschitz. Here we use the linearity of the surplus function $v_{i}(p)$ over prices $p$ to provide an alternative proof for the existence of directional derivatives in our context. Moreover, it relates the directional derivatives of the surplus function $v_{i}(p)$ to the directional derivatives of the objective function $v_{i}(S, p)$.

Lemma 5. For any given price vector $p \in R_{+}^{n}$ and any direction $w \in R_{+}^{n}$, there exists $S \in D_{i}(p)$ such that

$$
v_{i}^{\prime}(p ; w)=v_{i}^{\prime}(S, p ; w)=-<e(S), w>
$$

Proof. Consider the function family

$$
\left\{f_{i}(S, t)=: v_{i}(S, p+t w), S \subset N\right\}
$$

each of which is linear with respect to $t$. There are only a finite number of intersections (excluding intersections formed by identical lines). Assume that $t_{0}$ is the smallest positive number such that there is an intersection at this point. Therefore, there exists $S \subset N$ such that for any $S^{\prime} \subset N$ either $f_{i}\left(S^{\prime}, t\right)=f_{i}(S, t)$ on $\left[0, t_{0}\right]$; or, $f_{i}\left(S^{\prime}, t\right)<f_{i}(S, t)$ on $\left[0, t_{0}\right]$. Expressed in another way, we can say that

$$
S \in D_{i}(p+t w) \text { for } t \in\left[0, t_{0}\right] .
$$

Thus,

$$
v_{i}(p+t w)=v_{i}(S, p+t w), \quad t \in\left[0, t_{0}\right]
$$

Hence,

$$
v_{i}^{\prime}(p ; w)=\lim _{t \rightarrow 0^{+}} \frac{v_{i}(S, p+t w)-v_{i}(S, p)}{t}=v_{i}^{\prime}(S, p ; w) .
$$

This completes the proof.

Our next result shows that the sub-differential of the surplus function $v_{i}(p)$ coincides with the convex hull of the demand function $D_{i}(p)$. This makes it very easy to compute the sub-differential. But the major advantage of it is that we can apply those results in the nonsmooth analysis to analyzing our economic model. This makes our analysis much easier than the analysis directly working on the demand functions that are typically interdependent with each other, as shown in Examples 3 and 4. Lemma 6 is a direct application of Theorem 5 in Aubin and Cellina (1984, pp.36).

Lemma 6 (Upper Envelope Theorem). For any $p \in R_{+}^{n}$, we have

$$
\partial v_{i}(p)=-c o\left(D_{i}(p)\right) .
$$

Proof. Define $F: R_{+}^{n} \rightarrow R^{2^{n}}$ by

$$
F(p)=\left(v_{i}\left(S_{1}, p\right), v_{i}\left(S_{2}, p\right), \cdots, v_{i}\left(S_{2^{n}}, p\right)\right) .
$$


Note that each component of $F(p)$ is differentiable. Also note that

$$
v_{i}(p)=\max _{1 \leq j \leq 2^{n}} F_{j}(p)
$$

and

$$
D_{i}(p)=\left\{S_{j} \in \Sigma: v_{i}\left(S_{j}, p\right)=v_{i}(p)\right\} .
$$

Then it follows from Theorem 5 in Aubin and Cellina (1984, pp.36) that

$$
\partial v_{i}(p)=\overline{c o}\left\{\cup_{S_{j} \in D_{i}(p)} \nabla v_{i}\left(S_{j}, p\right)\right\},
$$

where $\overline{c o}(Z)$ denotes the closed convex hull of a set $Z$. Thus, we have that

$$
\partial v_{i}(p)=\overline{c o}\left\{-e(S): S \in D_{i}(p)\right\}=-c o\left(D_{i}(p)\right)
$$

since $\operatorname{co}\left(D_{i}(p)\right)$ is closed.

Some useful properties about the demand sets may be derived from Lemma 6 . For example, since $\partial v_{i}$ is maximal monotone, this implies that $\left\langle e(S)-e(T), p-q>\leq 0\right.$ for $S \in D_{i}(p)$ and $T \in D_{i}(q)$. This is quite similar to the downward sloping property of the single-valued demand function for divisible goods. Since $\partial v_{i}$ is upper semicontinuous, this implies that for any neighborhood $\mathcal{Z}$ of $\operatorname{co}\left(D_{i}(p)\right), p \in R_{+}^{n}$, there exists a neighborhood $\mathcal{Q}$ of $p$ such that $\operatorname{co}\left(D_{i}(q)\right) \subset \mathcal{Z}$ for all $q \in \mathcal{Q}$. These results are useful for their own sake and follow easily from the properties of the sub-differential of a regular function. Note that $D_{i}(p)$ is still monotone but not necessarily maximal monotone.

\section{$5 \quad$ Walrasian Equilibrium}

Let $W=\left\{p \in R_{+}^{n}: V(p) \leq V(q), \forall q \in R_{+}^{n}\right\}$. The set $W$ is the set of minimizers of the function $V(\cdot)$. Since $V(\cdot)$ is convex, $W$ is the set of globle minimizers of $V(\cdot)$. It is a nonempty, convex, and compact set. Next we show that in an economy that does not have a Walrasian equilibrium there exists at least one $D_{i}(p)$ that must contain more than one element in it for any $p$ in $W$.

Lemma 7. The function $v_{i}(p)$ is Gâteaux differentiable iff $D_{i}(p)$ is a singleton.

Proof. If $v_{i}(p)$ is differentiable, then $\partial v_{i}(p)=\left\{\nabla v_{i}(p)\right\}$. It follows from Lemma 6 that $D_{i}(p)$ is a singleton. Now, assume $D_{i}(p)$ is a singleton, Lemma 4 shows that for all directions $w \in R_{+}^{n}$, we have

$$
v_{i}^{\prime}(p ; w)=v_{i}^{\prime}(S, p ; w)=-<e(S), w>,
$$

where $\{S\}=D_{i}(p)$. This implies that $v_{i}(p)$ is Gâteaux differentiable. ${ }^{7}$

Theorem 8. Let $p \in W$. Suppose that the set-valued demand functions $D_{i}(p)$ are all singleton for all $i \in M$. Then a Walrasian equilibrium exists.

Proof. Lemma 7 shows that the functions $v_{i}(p)$ and $V(p)$ are differentable. Thus,

$$
\nabla V(p)=\left[\begin{array}{c}
1 \\
\vdots \\
1
\end{array}\right]+\sum_{i \in M} \nabla v_{i}(p)
$$

\footnotetext{
${ }^{7}$ Alternatively, it follows from Lemma 6 that a singleton $D_{i}(p)$ implies that $\partial v_{i}(p)$ is a singleton. Since $v_{i}(p)$ is continuous, it follows that it is Gâteaux differentiable.
} 
Since $p \in W$, this implies that $\nabla V(p)=0$ and then, by the Envelope Theorem,

$$
0=\left[\begin{array}{c}
1 \\
\vdots \\
1
\end{array}\right]-\sum_{i \in M}\left\{e\left(S_{i}\right): S_{i} \in D_{i}(p)\right\}
$$

This completes the proof.

Theorem 9. Suppose that the set $W$ of minimizers of the function $V(p)$ is of full dimension of $n$. Then a Walrasian equilibrium exists.

Proof. The set $W$ is a convex compact polyhedron. Since it is of full dimension and connected, there exists an open subset $U \subset W$ on which each $v_{i}(\cdot)$ is differentiable. Thus, it follows from Lemma 7 and Theorem 8 that a Walrasian equilibrium exists.

Thus, if an economy $\mathcal{E}$ does not have a Walrasian equilibrium, the set $W$ of minimizers of the function $V(p)$ must be a convex compact body of dimension at most $n-1$. Moreover, at each $p \in W$, there exists at least one demand function that contains at least two elements in it.

As shown above, the existence of a Walrasian equilibrium is closely tied up with the differentiation of the surplus functions $v_{i}(p), i \in M$. But there are economies such that a Walrasian equilibrium exists at which $v_{i}(p)$ may fail to be differentiable.

Example 10. Let $\epsilon_{1}=\epsilon_{2}=0$, and $\epsilon=\frac{1}{2}$ in Example 3. Let $p^{* * *}=\left(3,3 \frac{1}{2}, 3\right)$. Then we have

$$
D_{j}\left(p^{* * *}\right)=\{\{1\},\{3\},\{1,3\},\{1,2\}\} \text { and } D_{i}\left(p^{* * *}\right)=\{\{1\},\{3\},\{2,3\},\{1,3\}\} .
$$

Thus the price vector $p^{* * *}$ is Walrasian, with $V\left(p^{* * *}\right)=11 \frac{1}{2}=V$. It follows from Lemma 7 that both $v_{j}\left(p^{* * *}\right)$ and $v_{i}\left(p^{* * *}\right)$ are not differentiable at $p^{* * *}$.

\subsection{Lottery and Sunspot Equilibrium}

We now follow Garratt and Qin (1997) to introduce lotteries into our economy. Let $\Delta(\Sigma)$ denote the set of all probability distributions over $\Sigma$. Then a personalized lottery for an agent $i$ is represented by an element $\sigma_{i}$ in $\Delta(\Sigma)$. A lottery allocation $\sigma$ is an $m$-tuple $\sigma=\left(\sigma_{1}, \sigma_{2}, \cdots, \sigma_{m}\right)$ in $(\Delta(\Sigma))^{m}$.

Given a price vector $p \in R_{+}^{n}$, define $p(S)=<p, e(S)>$. Define $\tilde{v}_{i}: \Delta(\Sigma) \times R_{+}^{n} \rightarrow R$ by

$$
\tilde{v}_{i}\left(\sigma_{i}, p\right)=\sum_{S \in \Sigma} \sigma_{i}(S)\left[u_{i}(S)-p(S)\right]
$$

and $\tilde{D}_{i}: R_{+}^{n} \rightarrow \Delta(\Sigma)$ by

$$
\tilde{D}_{i}(p)=\left\{\sigma_{i} \in \Delta(\Sigma): \tilde{v}_{i}\left(\sigma_{i}, p\right) \geq \tilde{v}_{i}\left(\sigma_{i}^{\prime}, p\right), \forall \sigma_{i}^{\prime} \in \Delta(\Sigma)\right\} .
$$

Let $\mathcal{P}$ be the set of all partitions $\left(X_{1}, X_{2}, \cdots, X_{m}\right)$ of the set of objects $N$. Let $\Delta(\mathcal{P})$ denote the set of all probability distributions over $\mathcal{P}$. An element in $\Delta(\mathcal{P})$ represents a joint lottery for the economy. Given a joint lottery $\eta \in \Delta(\mathcal{P})$, it induces a lottery allocation $\sigma(\eta)=\left(\sigma_{1}(\eta), \sigma_{2}(\eta), \cdots, \sigma_{m}(\eta)\right)$ as follows

$$
\sigma_{i}(\eta)(T)=\sum_{\mathcal{S} \in \mathcal{P}: \mathcal{S}_{i}=T} \eta(T)
$$


for all $T \in \Sigma$ and all $i \in M$. The lottery allocation $\sigma(\eta)$ thus defined is the marginal distributions of the joint lottery $\eta$, a feasibility condition originated by Garratt (1995).

A lottery equilibrium $(p, \sigma)$ consists of a lottery allocation $\sigma=\left(\sigma_{1}, \sigma_{2}, \cdots, \sigma_{m}\right)$ and a price vector vector ${ }^{8} p \in R_{+}^{n}$ such that $\sigma_{i} \in \tilde{D}_{i}(p)$ for all $i \in M$, and there exists a joint lottery $\eta \in \Delta(\mathcal{P})$ such that $\sigma$ is induced by $\eta$.

Clearly, a Walrasian equilibrium is also a lottery equilibrium. The question of interest is if there exists a lottery equilibrium in an economy where a Walrasian equilibrium does not exist, a question that motivates the introduction of lotteries in Prescott and Townsend (1984) for economies with private information. The following result provides a negative result.

Theorem 11. There exists a lottery equilibrium in the economy $\mathcal{E}$ iff there exists a Walrasian equilibrium.

Proof. As we noted before, the existence of a Walrasian equilibrium implies the existence of a lottery equilibrium. So we need to show that if a Walrasian does not exist, a lottery equilibrium does not exist either. To show this, note first that for all $S_{1}, S_{2} \in D_{i}(p), u_{i}\left(S_{1}\right)-p\left(S_{1}\right)=u_{i}\left(S_{2}\right)-p\left(S_{2}\right) \geq$ $u_{i}(S)-p(S)$ for all $S \in \Sigma$. Secondly, note that for any $\sigma_{i}^{\prime} \in \tilde{D}_{i}(p), \sigma_{i}^{\prime}(S)>0$ for $S \in \Sigma$ only if $S \in D_{i}(p)$.

Now suppose that there is a lottery equilibrium $(p ; \sigma)$. By definition, there exists a joint lottery $\eta$ such that $\sigma$ is induced by $\eta$. Let $X \in \mathcal{P}$ be a partition of $N$ such that $\eta(X)>0$. Note that

$$
\sigma_{i}(\eta)\left(X_{i}\right)=\sum_{Y \in \mathcal{P}: Y_{i}=X_{i}} \eta(Y) \geq \eta(X)>0
$$

This implies that $\sigma_{i}\left(X_{i}\right)>0$ since $\sigma_{i}\left(X_{i}\right)=\sigma_{i}(\eta)\left(X_{i}\right)$. This shows that $X_{i} \in D_{i}(p)$ for all $i \in M$. But since $X$ is a partition, this implies that a Walrasian equilibrium exists, a contradiction.

Theorem 11 shows that an introduction of lotteries does not introduce a new equilibrium beyond a Walrasian equilibrium. This is quite similar to Garratt (1995) who studied an Arrow-Debreu general equilibrium economy with indivisible goods. He showed that a lottery equilibrium is either a Walrasian or a sunspot equilibrium. But, there are Walrasian and sunspot equilibria that are not lottery equilibria.

In contrast, Garratt et al (2001) studied an Arrow-Debreu general equilibrium economy with a finite number of consumers and a continuous sunspot variable and showed that lottery and sunspot equilibrium are equivalent in the equilibrium allocations. But if sunspot variable is finite, Garratt, Keister and Shell (2001) showed that some substantial differences exist between the two notions. They have to introduce the notion of constrained lotteries in order to bridge the gap of the two. One may wonder if lottery and sunspot equilibrium are different in our economic model. Next we will investigate this issue.

Let $(\Omega, \mathcal{F}, \pi)$ be a probability space, where $\Omega$ is a finite set with $K$ elements, $\mathcal{F}$ is the set of all subsets of $\Omega$, and $\pi$ is a probability measure on $\Omega$.

Let $\bar{\Sigma}=\{e(S): S \in \Sigma\}$. Let $\Lambda$ be the set of functions $x: \Omega \rightarrow \bar{\Sigma}$. Prices for the indivisible goods are given by a function $\bar{p}: \Omega \rightarrow R_{+}^{n} \cdot{ }^{9}$ Let $\bar{P}$ denote the set of all such price functions.

\footnotetext{
${ }^{8}$ Precisely the price vector $p$ should be in the space $R_{+}^{2^{n}}$, satisfying the nonarbitrage condition: $p(e(S))=\sum_{j \in S} p_{j}$ for all $S \in \Sigma$. Since our price system is linear and satisfy $p(S)=\sum_{j \in S} p_{j}$ for all $S \in \Sigma$, the two are equivalent.

${ }^{9}$ As in Garratt et al (2001), the prices $\bar{p}$ here are the actual contingent-commodity prices not probability-adjusted prices.
} 
Define $\bar{v}_{i}: \Lambda \times \bar{P} \rightarrow R$ by

$$
\bar{v}_{i}\left(x_{i}, \bar{p}\right)=\sum_{\omega} \pi(\omega) u_{i}\left(x_{i}(\omega)\right)-\sum_{\omega}<\bar{p}(\omega), x_{i}(\omega)>.
$$

Define $\bar{D}_{i}: \bar{P} \rightarrow \Lambda$ by $\bar{D}_{i}(\bar{p})=\left\{x_{i} \in \Lambda: \bar{v}_{i}\left(x_{i}, \bar{p}\right) \geq \bar{v}_{i}\left(y_{i}, \bar{p}\right), \forall y_{i} \in \Lambda\right\}$. Let $\bar{v}_{i}(\bar{p})=\bar{v}_{i}\left(x_{i}, \bar{p}\right)$ for $x_{i} \in \bar{D}_{i}(\bar{p})$. One may follow the proof of Lemma 1 to show that $\bar{v}_{i}(\bar{p})$ is convex and globally Lipschitz on $\bar{P}$.

An allocation $(x(\omega))_{\omega \in \Omega}=\left(\left(x_{1}(\omega), x_{2}(\omega), \cdots, x_{m}(\omega)\right)_{\omega \in \Omega}\right.$ is feasible if it satisfies the following

$$
\forall \omega \sum_{i \in M} x_{i}(\omega)=\left[\begin{array}{c}
1 \\
\vdots \\
1
\end{array}\right] .
$$

A sunspot equilibrium consists of a price function $\bar{p} \in \bar{P}$ and a feasible allocation $(x(\omega))_{\omega \in \Omega}$ such that $x_{i} \in \bar{D}_{i}(\bar{p})$ for all $i \in M$.

Let $\bar{\Lambda}$ denote the set of all feasible allocations. Let

$$
\bar{V}=\max _{x \in \bar{\Lambda}} \sum_{i \in M} \sum_{\omega} \pi(\omega) u_{i}\left(x_{i}(\omega)\right)
$$

A feasible allocation $x$ is optimal if it is a solution to the above maximazition problem in $\bar{V}$.

Define

$$
\bar{V}(\bar{p})=\sum_{\omega}<\bar{p}(\omega), N>+\sum_{i \in M} \bar{v}_{i}(\bar{p})
$$

Lemma 12. The following hold

1. $\bar{V}=V$.

2. $\bar{V}(p) \geq \bar{V}$ for all $p \in \bar{P}$.

3. Prices $p \in \bar{P}$ are sunspot equilibrium prices iff $\bar{V}(p)=\bar{V}$.

Proof. Note that

$$
\bar{V}=\max _{x \in \bar{X}} \sum_{\omega} \pi(\omega) \sum_{i \in M} u_{i}\left(x_{i}(\omega)\right)=\sum_{\omega} \pi(\omega) V=V
$$

The rest proofs follow from the idea in the proof of Lemma 2 and thus omitted.

The term $V$ is the total social welfare. Lemma 12(1) shows that sunspots do not increase the total social welfare to the original economy. Lemma12(2) and (3) are the first and second welfare theorems: A sunspot equilibrium allocation is optimal; If a sunspot equilibrium exists, every optimal allocation is supported by a sunspot equilibrium price vector.

Lemma 13. Let $\bar{p} \in \bar{P}$. For any $x_{i} \in \bar{D}_{i}(\bar{p}), x_{i}(\omega) \in\left\{e(S): S \in D_{i}\left(\frac{1}{\pi(\omega)} \bar{p}(\omega)\right)\right\}$ for all $\omega$ such that $\pi(\omega)>0$. 
Proof. By definition,

$$
\begin{aligned}
\bar{v}_{i}\left(\left(x_{i}, \bar{p}\right)\right) & =\sum_{\omega} \pi(\omega) u_{i}\left(x_{i}(\omega)\right)-\sum_{\omega}<\bar{p}(\omega), x_{i}(\omega)> \\
& =\sum_{\omega} \pi(\omega)\left[u_{i}\left(x_{i}(\omega)\right)-<\frac{1}{\pi(\omega)} \bar{p}(\omega), x_{i}(\omega)>\right]
\end{aligned}
$$

Since $\pi(\omega)>0, x_{i} \in \bar{D}_{i}(\bar{p})$ implies that $x_{i}(\omega)=e(S)$ for some $S \in D_{i}\left(\frac{1}{\pi(\omega)} \bar{p}(\omega)\right)$. This completes the proof.

Theorem 14. A Walrasian equilibrium exists iff a sunspot equilibrium exists.

Proof. Let $(X, p)$ be a Walrasian equilibrium. Let $\bar{p}(\omega)=\pi(\omega) p$ and $x_{i}(\omega)=e\left(X_{i}\right), \forall \omega \in \Omega$. Since

$$
\bar{v}_{i}\left(\left(x_{i}, \bar{p}\right)\right)=\sum_{\omega} \pi(\omega)\left[u_{i}\left(x_{i}(\omega)\right)-<\frac{1}{\pi(\omega)} \bar{p}(\omega), x_{i}(\omega)>\right],
$$

it follows from $X_{i} \in D_{i}(p)$ that $\left(x_{i}(\omega)\right)_{\Omega} \in \bar{D}_{i}(\bar{p})$.

Now suppose $(x, \bar{p})$ is a sunspot equilibrium. Then Lemma 13 shows that one can construct a Walrasian equilibrium from it. This completes the proof.

Theorems 11 and 14 together show that a lottery equilibrium exists iff a sunspot equilibrium exists. As a result, Walrasian equilibrium, lottery equilibrium and sunspot equilibrium are all equivalent for our model. Under the gross substitutes condition of Kelso and Crawford (1982), lottery and sunspot equilibrium both exist in the economy $\mathcal{E}$. Clearly, there are also economies that may not have lottery and sunspot equilibria. In particular, lotteries and sunspot variables do not provide new equilibria for economies that do not have a Walrasian equilibrium.

\section{Stability}

In this section, we first answer the question how objects may be allocated when prices are fixed at some level. Then we discuss a price adjustment process in continuous time. Our process may be considered as a tatonnement process as in an English auction if trade of objects can be made only by the end of the process. It may also be considered as a trading process if trade is allowed during the process.

\subsection{Edgeworth trading hypothesis}

A Walrasian equilibrium exists if a feasible allocation $X=\left(X_{0}, X_{1}, X_{2}, \cdots, X_{m}\right)$ and a price vector $p$ can be found such that $X_{i}$ is in agent $i$ 's demand function $D_{i}(p)$ for all $i \in M$ and $<e\left(X_{0}\right), p>=0$. This definition of an equilibrium is standard throughout the literature. But it may have a potential problem if a demand function turns out to be a set-valued mapping. Consider Example 10. At prices $p^{* * *}=\left(3,3 \frac{1}{2}, 3\right)$. The demand functions for agents $j$ and $i$ are as follows

$$
D_{j}\left(p^{* * *}\right)=\{\{1\},\{3\},\{1,3\},\{1,2\}\} \text { and } D_{i}\left(p^{* * *}\right)=\{\{1\},\{3\},\{2,3\},\{1,3\}\} .
$$

There are two Walrasian equilibrium allocations. One is $\left(X_{0}^{1}, X_{j}^{1}, X_{i}^{1}\right)=(\emptyset,\{1\},\{2,3\})$ and the other $\left(X_{0}^{2}, X_{j}^{2}, X_{i}^{2}\right)=(\emptyset,\{1,2\},\{3\})$. Note that there are other bundles in $D_{j}\left(p^{* * *}\right)$ and $D_{i}\left(p^{* * *}\right)$ that are also available. This means that there are other object bundles that provide agents $i$ and $j$ the same consumer surplus as the equilibrium bundles. Why do we believe that the two Walrasian 
equilibrium allocations are more reasonable than the others, say, $(\{2\},\{1\},\{3\})$ or $(\{2\},\{3\},\{1\})$, where object 2 is the unallocated object? Note that these two alternatives are equivalent to the two equilibrium allocations with respect to agents $i$ 's and $j$ 's self-interests. There must be an underlying assumption in the definition of a Walrasian equilibrium under which only the Walrasian allocations are chosen. We believe that it is the Edgeworth trading hypothesis discussed in Fisher (1983): "[T]rade will take place if and only if there is some group of people who can all be made better off by trading among themselves at the current prices." This is the notion of the core. Now assume that agent 0 owns all objects and has zero utility over any object bundle. The Edgeworth trading hypothesis implies that "markets are sufficient well organized" (Fisher (1983)) such that objects are allocated to those agents such that no new trade can be made possible to make agents, including agent 0 , better off. This is the principle of "Pareto efficiency", contingent upon the fixed price level. When it applies to our example above, only the two Walrasian allocations will be chosen. This is exactly what is needed. The question is how this can be formalized for those situations where the fixed prices may not be Walrasian.

Let $p \in R_{+}^{n}$ be any price vector. Let $\mathcal{X}$ denote the set of all feasible allocations in the economy $\mathcal{E}$. Suppose that agent 0 owns the objects $N$ and has zero utility over any object bundle. Since $p$ is now fixed and arbitrary, one should not expect that the market clearing condition can be satisfied. To apply the Edgeworth trading hypothesis to our model, we consider the following maximization problem:

$$
V^{*}(p)=\max _{X \in \mathcal{X}^{*}} \sum_{i \in M}<e\left(X_{i}\right), p>+\sum_{i \in M} v_{i}\left(X_{i}, p\right)
$$

where $\mathcal{X}^{*}=\left\{X \in \mathcal{X}: v_{i}\left(X_{i}, p\right) \geq 0, \forall i \in M\right\}$. The set $\mathcal{X}^{*}$ is all feasible allocations that are individually rational (contingent upon the prices) for all agents in $M$. The first term $<\sum_{i \in M} e\left(X_{i}\right), p>$ in $V^{*}(p)$ is the total revenue for agent 0 of those objects "purchased" by agents in $M$ at the prices $p$ and the second term $\sum_{i \in M} v_{i}\left(X_{i}, p\right)$ is the total social surplus to all agents in $M$. Suppose that $X$ is a feasible allocation that is a solution to the problem $V^{*}(p)$. Then there exist no other feasible allocations that can make all agents better off. Since $\mathcal{X}^{*}$ is a finite set, a solution always exists to the maximization problem $V^{*}(p)$ for any given $p$. Let

$$
\mathcal{X}^{*}(p)=\left\{X \in \mathcal{X}^{*}: V^{*}(p)=\sum_{i \in M}<e\left(X_{i}\right), p>+\sum_{i \in M} v_{i}\left(X_{i}, p\right)\right\}
$$

denote the set of all solutions to the problem $V^{*}(p)$. Let $\Xi: R_{+}^{n} \rightarrow X$ denote a market outcome in allocations of objects. We say that $\Xi$ satisfies the Edgeworth trading hypothesis if for all prices $p \in R_{+}^{n}$,

$$
\Xi(p) \in \mathcal{X}^{*}(p)
$$

Clearly, $V^{*}(p) \leq V$ for all prices $p \in R_{+}^{n}$. If $p$ is Walrasian, then $V^{*}(p)=V$ and $\mathcal{X}^{*}(p)$ coincides with the set of all Walrasian equilibrium allocations. So the map $p \mapsto \Xi(p)$ is indeed the desired one.

\subsection{Price Adjustment Process}

We now provide a price adjustment process that can be applied to an economy that may not have a Walrasian equilibrium. Of course, our process also works well for an economy that has a Walrasian equilibrium.

Let

$$
\Gamma=\left\{t_{0}, t_{1}, \cdots, t_{\mu-1}, t_{\mu}\right\}
$$


be any partition of $[0, b]$, where $t_{0}=0$ and $t_{\mu}=b$. We are interested in the Classic Euler iterative scheme:

$$
\frac{p\left(t_{j}\right)-p\left(t_{j-1}\right)}{t_{j}-t_{j-1}}-f\left(p\left(t_{j-1}\right)\right)=0, j=1,2, \cdots, \mu-1, \mu,
$$

where $p(0)=p_{0} \in \operatorname{Dom}(V(\cdot))$, and

$$
f\left(p\left(t_{j-1}\right)\right) \in \sum_{i} \operatorname{co}\left(D_{i}\left(p\left(t_{j-1}\right)\right)\right)-\left[\begin{array}{c}
1 \\
\vdots \\
1
\end{array}\right] .
$$

Therefore, $f\left(p\left(t_{j-1}\right)\right)$ is an excess demand bundle.

The timing of this price iterative scheme may be illustrated in Figure 1.

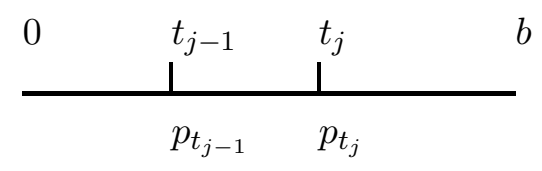

Figure 1. An Euler Price Iterative Process

A Tatonnement Process: It may be helpful to have a stylized story to illustrate this price adjustment process. Imagine that there are $m$ computer terminals that are online. At time 0 , all computer terminals are connected to a central network. Each agent is assigned with one computer terminal. On each terminal, the initial prices $p\left(t_{0}\right)$ and the objects that are for sale are posted on the terminal. When the central network announces that the process begins, each agent starts to place his market order of an object bundle he is willing to buy at the prices $p\left(t_{0}\right)$. Based on the new information of the market orders, the central network adjusts the prices according to (8). Then the process moves to period 1 and the new adjusted prices $p\left(t_{1}\right)$ become the new market prices and are posted on each terminal. Agents then place their market orders for the new posted prices. This process continues until the distance of the two adjacent price vectors are no larger than an $\epsilon$, where $\epsilon$ can be chosen to be small. After that, players are asked to play an implementation game presented in Section 7 that guarantees that objects will be allocated according to $\Xi\left(p\left(t^{*}\right)\right.$, where $t^{*}$ is the time that the process stops.

To study the property of global stability of the dynamics (8), we consider a more general price adjustment process, which is a version of the process of (8) with deterministic noises:

$$
\frac{p\left(t_{j}\right)-p\left(t_{j-1}\right)}{t_{j}-t_{j-1}}-f\left(p\left(t_{j-1}\right)\right)=u\left(t_{j-1}\right), j=1,2, \cdots, \mu-1, \mu,
$$

where $f\left(p\left(t_{j-1}\right)\right)$ is as in $(8)$, and the noise term $u:[0, b] \rightarrow R^{n}$ is a measurable function on $[0, b]$ satisfying

$$
\|u\|_{L^{2}\left([0, b], R^{n}\right)}=\left(\int_{0}^{b}\|u\|^{2} d t\right)^{\frac{1}{2}}<\infty .
$$

Thus, given a partition $\Gamma$ and a selection $f$, we obtain a sequence of prices $p\left(t_{0}\right), \cdots, p\left(t_{j-1}\right)$, $p\left(t_{j}\right), \cdots, p\left(t_{\mu}\right)$. Such a sequence is called the Euler polygonal arc on $[0, b]$, denoted by $p_{\Gamma}^{f}$, which of course depends on the noise term $u$ and the initial state $p_{0}$.

This construction of Euler polygonal arcs is quite arbitrary. Since each $D_{i}(\cdot)$ is a set-valued mapping, there are a family of Euler polygonal arcs, denoted by $p_{\Gamma}$, for a given partition and an 
initial state $p_{0}$. Surprisingly, all these Euler polygonal arcs have the common limit that is unique as the number of partition points $\mu$ approaches $\infty$, as shown in Theorem 15.

The diameter $\mu_{\Gamma}$ of the partition $\Gamma$ is defined by $\mu_{\pi}=\max \left\{t_{j}-t_{j-1}: j=1,2, \cdots \mu\right\}$. Given a selection $f$, an Euler solution to (9) is defined to be any arc which is the uniform limit of Euler polygonal $\operatorname{arcs} p_{\Gamma}^{f}$ as the diameter $\mu_{\Gamma}$ approaches 0 (i.e., the corresponding $\mu$ approaches $\infty$ ). An Euler solution is also called an Euler price adjustment process.

Theorem 15. There exists a unique Euler price adjustment process $p(t)$ on $[0, b]$ to $(9)$ that is the only absolutely continuous function $p:[0, b] \rightarrow R^{n}$, together with its derivative with respect to $t$, satisfying the following differential inclusion

$$
\frac{d p(t)}{d t} \in \sum_{i} c o\left(D_{i}(p(t))\right)-\left[\begin{array}{c}
1 \\
\vdots \\
1
\end{array}\right]+u(t), p(0)=p_{0} \in \operatorname{Dom}(V(\cdot))
$$

where $u(t)$ is a measurable function on $[0, b]$ satisfying ${ }^{10}$

$$
\|u\|_{L^{2}\left([0, b], R^{n}\right)}=\left(\int_{0}^{b}\|u\|^{2} d t\right)^{\frac{1}{2}}<\infty .
$$

Before the proof, we need some definitions from the control theory (Clarke et al (1988)). Consider a differential inclusion

$$
\frac{d x(t)}{d t} \in F(t, x(t)) a . e ., t \in[a, b]
$$

where $F: R \times R^{n} \rightarrow R^{n}$ is a set-valued mapping. An absolutely continuous function $x:[a, b] \rightarrow R^{n}$ is called an arc on $[a, b]$. A solution to (10) or a trajectory of $F$ is an absolutely continuous function $x:[a, b] \rightarrow R^{n}$ which, together with its derivative $\frac{d x(t)}{d t}$ with respect to $t$, satisfies (10).

The set-valued mapping $F$ satisfies the Standing Hypotheses if the following hold:

(a). For every $(t, x), F(t, x)$ is a nonempty, compact, convex set.

(b). $F$ is upper semicontinuous.

(c). For some positive constants $\gamma$ and $c$, and for all $(t, x)$,

$$
v \in F(t, x) \Longrightarrow\|v\| \leq \gamma\|x\|+c .
$$

Let $f$ be any selection of $F$, let $x$ be any Euler solution on $[a, b]$ of $\frac{d x(t)}{d t}=f(t, x(t)), x(a)=x_{0}$. Then Corollary 1.12 in Clarke et al (1988, pp.186) shows that $x$ is a trajectory of $F$ on $[a, b]$. Even though the Euler solution $x$ is a trajectory of $F$, this does not mean that it satisfies $\frac{d x(t)}{d t}=f(t, x(t))$; see Clarke et al (1988) for examples. Also note that an Euler solution is not necessarily unique for a given selection $f$. Since $F$ is a set-valued map, one should not in general expect that there exists a unique Euler solution to (10) for a given initial point $x_{0}$. Our unique result in Theorem 15 is an application of Corollary 1.12 in Clarke et al (1988, pp.186) and a result in Yotrutani (1978). The reason why we can obtain a unique Euler solution of $(9)$ is largely due to the fact that $V(\cdot)$ is

\footnotetext{
${ }^{10}$ This condition is automatically satisfied in our context, since each market order is always bounded by $\left[\begin{array}{c}1 \\ \vdots \\ 1\end{array}\right]$.
} 
convex and continuous. So one can apply the result in Yotrutani (1978).

Proof. Define $F: R_{+}^{n} \rightarrow R^{n}$ by

$$
F(p)=-\partial V(p)+u
$$

Since $V$ is convex and regular by Lemma 1, it is known that $\partial V$ is a nonempty, convex, and compact set. Further, it is upper semicontinuous. Therefore, $F$ satisfies the Standing Hypotheses. Let $f$ be any selection of $-\partial V(p)$. By Theorem 1.7 and Corollary 1.12 in Clarke et al (1988, pp.183\&186), there exists an Euler solution $p$ on $[0, b]$ of the problem

$$
\frac{d p(t)}{d t}=f(p(t))+u(t), p(0)=p_{0} \in \operatorname{Dom}(V(\cdot))
$$

that is a trajectory of $F$ on $[0, b]$.

Since the function $V(\cdot)$ is convex and continuous by Lemma $1, \partial V$ is a maximal monotone operator. It is known from Yotrutani (1978) that there is a unique absolutely continuous function $t \mapsto p(t)$ on $[0, b]$ that, together with its derivative with respect to $t$, satisfies

$$
\frac{d p(t)}{d t} \in-\partial V(p(t))+u(t), p(0)=p_{0} \in \operatorname{Dom}(V(\cdot)) .
$$

Therefore, the Euler solution $p(t)$ defined on $[0, b]$ must be unique since the Euler solution is an absolutely continuous function that satisfies (11). Now it follows from the Upper Envelope Theorem that

$$
-\partial V(p)=\sum_{i} c o\left(D_{i}(p)\right)-\left[\begin{array}{c}
1 \\
\vdots \\
1
\end{array}\right] .
$$

This completes the proof.

Let $p\left(u, p_{0}\right)(\cdot)$ denote the unique Euler price adjustment process defined on $[0, b]$ that is identified in Theorem 15, which depends on the noise term $u$ and the initial state $p_{0} \in \operatorname{Dom}(V(\cdot))$. We are interested in the behavior of this process when the noise term $u$ approaches 0 . Let $p\left(0, p_{0}\right)(t)$ be the Euler price adjustment process defined on $[0, b]$ to $(8)$. We first examine the convergence property of $p\left(0, p_{0}\right)(\cdot)$.

Theorem 16. The Euler price adjustment process $p\left(0, p_{0}\right)(\cdot)$ on $[0, b]$ of $(8)$ reaches an equilibrium in finite time. Precisely, for any $p_{0} \in \operatorname{Dom}(V(\cdot))$, there exist $p^{*} \in W$, i.e., a minimizer of $V(p)$ in $R_{+}^{n}$, and a strictly positive constant $\alpha$ such that

$$
t^{*} \leq \frac{\left(V\left(p_{0}\right)-\min _{p} V(p)\right)}{\alpha^{2}}
$$

and

$$
\forall t \geq t^{*}, \quad V\left(p\left(0, p_{0}\right)(t)\right)=V\left(p^{*}\right)
$$

The principal idea in the proof of Theorem 16 is to divide $R_{+}^{n}$ into a set of subsets on each of which $\partial V(p)$ is a convex hull of sub-differentials of a set of linear functions (therefore the convex hull of a set of constant vectors) and the minimal norm of the convex hull is greater than 0 unless 
the subset consists of minimizers of $V(p)$. Hence $V(p(t))$ decreases with a minimal speed that is greater than 0 .

For any $m$-tuple $\mathcal{S}=\left(S_{1}, S_{2}, \cdots, S_{m}\right) \in \Sigma^{m}$, let

$$
v_{\mathcal{S}}(p)=\sum_{i=1}^{m} v_{i}\left(S_{i}, p\right) .
$$

Definition. For any $p \in R_{+}^{n}$, let

$$
\Phi(p)=\left\{\mathcal{S} \in \Sigma^{m}: v_{\mathcal{S}}(p)=\max _{\mathcal{S}^{\prime} \in \Sigma^{m}} v_{\mathcal{S}^{\prime}}(p)\right\}
$$

For any $T \subset \Sigma^{m}$, let

$$
\wp(T)=\left\{p \in R_{+}^{n}: \Phi(p)=T\right\}
$$

Proof of Theorem 16. Step 1. The following identity holds:

$$
V(p)=\sum_{j=1}^{n} p_{j}+\max _{\mathcal{S} \in \Sigma^{m}} v_{\mathcal{S}}(p) .
$$

Proof. On one hand, we have

$$
\max _{\mathcal{S} \in \Sigma^{m}} v_{\mathcal{S}}(p)=\max _{\left(S_{1}, S_{2}, \cdots, S_{m}\right) \in \Sigma^{m}} \sum_{i=1}^{m} v_{i}\left(S_{i}, p\right) .
$$

On the other hand,

$$
\sum_{i=1}^{m} v_{i}(p)=\sum_{i=1}^{m} \max _{S_{i} \subset N} v_{i}\left(S_{i}, p\right)
$$

Since the summation and the maximization are taken on finite sets, we can exchange the order of summation and maximization and conclude that the two sides of (12) are the same.

Step 2. Given any $T \in \Sigma^{m}, \partial V$ is constant on $\wp(T)$. That is, we have

$$
\partial V(p)=\partial V(q), \forall p, q \in \wp(T) .
$$

Proof. By Step 1 and maximization rule (as well as additin rule) of sub-differential (Theorem 5 in Aubin and Cellina (1984, pp.36)) we have

$$
\partial V(p)=\left[\begin{array}{c}
1 \\
\vdots \\
1
\end{array}\right]+\overline{c o}\left\{\partial v_{\mathcal{S}}(p): \mathcal{S} \in \Phi(p)\right\}
$$

Note that each $v_{\mathcal{S}}$ is linear and therefore $\partial v_{\mathcal{S}}(p)$ is constant, we conclude that the right side of the above equation is constant (set) too.

Step 2 indicates that $R_{+}^{n}$ is divided into at most $\operatorname{rank}\left(\Sigma^{m}\right)$ non-empty subsets (some $\wp(T)$ ) could be empty) such that, on each such subset, $\partial V(p)$ is a constant set with respect to $p$. Denote 
by $\operatorname{sub}(T)$ the sub-differential $\partial V(p)$ at any price vector $p \in \wp(T)$, we can categorize all $\wp(T)$ s into two categories $\Im_{0}$ and $\Im_{1}$ :

and

$$
\Im_{0}=\left\{\wp(T): T \subset \Sigma^{m} \text { and } 0 \in \operatorname{sub}(T)\right\}
$$

$$
\Im_{1}=\left\{\wp(T): T \subset \Sigma^{m} \text { and } 0 \notin \operatorname{sub}(T)\right\} .
$$

We also denote by $\Re_{0}$ and $\Re_{1}$ the union of sets in $\Im_{0}$ and the union of sets in $\Im_{1}$ respectively. Then, $\Re_{0}$ contains all minimum price vectors of $V(p)$, i.e., $\Re_{0}=W$, and $\Re_{1}$ is connected since $V(p)$ is convex. More importantly, since each sub-differential is closed, we have

$$
\text { inf } f_{p \in \Re_{1}, d \in \partial V(p)}\|d\|=\alpha>0 .
$$

Applying this to (4) in Aubin and Cellina (1984, pp.160), for any $t<\infty$, we have

$$
V(p(t))-V(p(0)) \leq-\int_{0}^{t}\left\|p^{\prime}\left(0, p_{0}\right)(\tau)\right\|^{2} d \tau \leq-\alpha^{2} \cdot t .
$$

Hence, if we let $t^{*}\left(p_{0}\right)$ be the earliest time for $p(t)$ to reach a minimum, we have

$$
t^{*}\left(p_{0}\right) \leq \frac{\left(V\left(p_{0}\right)-\min _{p} V(p)\right)}{\alpha^{2}} .
$$

Note that the function $t \mapsto V(p(t))$ is convex non-increasing, by Theorem 1 in Aubin and Cellina (1984, pp.159). This implies that once the process $\{p(t)\}$ reaches the minimum, it will stay thereafter. This completes the proof.

Theorem 16 is much stronger than Theorem 2 in Aubin and Cellina (1984, pp.160) which has shown that the trajectory of the gradient inclusion

$$
\frac{d p(t)}{d t} \in-\partial V(p(t)), p_{0} \in \operatorname{Dom}(V(\cdot))
$$

converges weakly to its asymptotic center which minimizes $V(\cdot)$ as $t \rightarrow \infty$. Theorem 16 shows that this can in fact happen in finite time. Moreover, the weakly convergence turns out to be the strong convergence in our case.

Proposition 3.3 in Hu and Papageorpiou (1998) shows that the function $u \mapsto p(u, p(0))$ is continuous. Therefore, $p(u, p(0)) \rightarrow p(0, p(0))$ as $u \rightarrow 0$ for any $p(0) \in \operatorname{Dom}(V(p))$. This, together with Theorems 15 and 16, shows that

Corollary 17. Assume that a Walrasian equilibrium exists. Then for any $p_{0} \in \operatorname{Dom}(V(p))$, there exist a Walrasian equilibrium price vector $p^{*}$ and a finite time $t^{*}$ such that the Euler price adjustment process of (8) converges to $p^{*}$ and $p(t) \in W$ for all $t \geq t^{*}$.

Proof. Theorem 16 and Lemma 2 show that there exists a finite $t^{*}$ such that $V(p(t))=V$ for all $t \geq t^{*}$. Then, by Lemma 2 again, the price vector $p(t)$ is Walrasian.

The following result is a direct result of Theorem 15. It shows that the Euler price adjustment process of (8) converges to $p^{*}$ for some $p^{*} \in W$ and stays there once it reaches there.

Corollary 18. Suppose that an economy $\mathcal{E}$ does not have a Walrasian equilibrium. Then, for any $p_{0} \in \operatorname{Dom}(V(p))$, there exist a price vector $p^{*} \in W$ and a finite time $t^{*}$ such that the Euler 
price adjustment process of (8) converges to $p^{*}$ at $t^{*}$ and $p(t) \in W$ for all $t \geq t^{*}$.

Therefore, we define a price vector $p \in W$ as a market equilibrium price vector. The set $W$ contains all market equilibrium price vectors. In an economy with a Walrasian equilibrium, there are no price vectors other than the Walrasian price vectors that have the property of global stability under the Euler price adjustment process of (8). In an economy without a Walrasian equilibrium, there are no price vectors other than the market equilibrium price vectors in $W$ that have the property of global stability under the Euler price adjustment process of (8). In an economy that has a Walrasian equilibrium, the market price vectors are nothing but the Walrasian equilibrium price vectors.

\subsection{Examples}

As we noted above, the demand functions for agents $j$ and $i$ in Example 10 at prices $p^{* * *}=\left(3,3 \frac{1}{2}, 3\right)$ are set-valued maps:

$$
D_{j}\left(p^{* * *}\right)=\{\{1\},\{3\},\{1,3\},\{1,2\}\} \text { and } D_{i}\left(p^{* * *}\right)=\{\{1\},\{3\},\{2,3\},\{1,3\}\} .
$$

Corollary 18 shows that starting from any initial price vector $p_{0} \in \operatorname{Dom}(V(\cdot))$, the price adjustment process of $(8)$ converges to the price vector $p^{* * *}$ in finite time and be stable at there. Moreover, there are no price vectors other than $p^{* * *}$ that have such a stability property. The map $\Xi^{*}\left(p^{* * *}\right)$ contains exactly the two optimal allocations.

The following is a special case of the general example used by Milgrom (2000) to show that an economy may not have a Walrasian equilibrium if even one agent has preferences that are complementary, and every other agent has preferences that are mutual substitutes. Walrasian equilibrium is not helpful in predicting what may be the outcome in this example. Because the salary-adjustment process in Kelso and Crawford (1982) and the English auctions in Gül and Stacchetti (2000) both depend on the gross substitutes condition, they are not applicable to this example. Milgrom (2000) showed that in the simultaneous ascending auction, the auction may end up with the prices that are at least 1.75 and 1.75 minus one increment. With the Euler polygonal arcs of (8), we end up with an outcome that is substantially different.

Example 19 (Milgrom (2000)). There are two objects $N=\{1,2\}$ and two agents $M=\{j, k\}$. The preferences are as follows:

$$
\begin{gathered}
u_{j}(\{1\})=1, \quad u_{j}(\{2\})=1, \quad u_{j}(\{1,2\})=3 \\
u_{k}(\{1\})=1 \frac{3}{4}, \quad u_{k}(\{2\})=1 \frac{3}{4}, \quad u_{k}(\{1,2\})=2 \frac{3}{4}
\end{gathered}
$$

Note that $V=3$ and $V(p)$ reaches its minimum at the prices $(1.5,1.5)$. According to Corollary 19 , the price vector $(1.5,1.5)$ is stable for the Euler price adjustment process of $(8)$. The allocation of objects is made according to $\Xi(1.5,1.5)=(\emptyset,\{1,2\}, \emptyset)$, where agent $j$ is allocated the bundle $\{1,2\}$. This is a reasonable allocation. It is optimal. Note that agent 0 extracts all economic rents 3 . The presence of complementary preferences benefits the seller. With the gross substitutes preferences, buyers typically enjoy positive surpluses at a Walrasian equilibrium.

Let us show how the Euler polygonal arcs perform under such a situation. This is also heplful for understanding those stability results in Corollaries 17 and 18 . We start with prices $(0,0)$ and the (constant) increment $\pi_{\Gamma}=0.1$ (i.e. the uniform partition with a diameter that equals 0.1 ). 
One can show that all possible Euler polygonal arcs (without randomization) of (8) will monotonely converge to the prices $(1.5,1.5)$ and then stay in the set

$$
\{(1.5,1.4),(1.4,1.5),(1.5,1.5),(1.6,1.5),(1.5,1.6)\} \text {. }
$$

Note that each element in this set is $\pi_{\Gamma}$-close to $(1.5,1.5)$. As the increment $\pi_{\Gamma}$ approaches zero, all Euler polygonal arcs converge to $(1.5,1.5)$ and stay at there.

One may think why the price vector $p^{\Delta}=(1.65,1.65)$ is not stable for $(8)$. At these prices, we have that

$$
D_{j}\left(p^{\Delta}\right)=\{\emptyset\}, D_{k}\left(p^{\Delta}\right)=\{\{1\},\{2\}\} .
$$

Starting with $(1.65,1.65)$, all possible Euler polygonal arcs (without randomization) generated by (8) will monotonely converge to $(1.55,1.55)$ and then stay in the set

$$
\{(1.45,1.45),(1.55,1.55),(1.45,1.55),(1.55,1.45)\} \text {. }
$$

Thus, all Euler polygonal arcs of (8) will move away from the prices $(1.65,1.65)$ and never come back to it. In fact, these results are by no means an accident but examples of a general result shown below.

Theorem 20. Let $p_{0} \in \operatorname{Dom}(V(\cdot))$ and $p\left(0, p_{0}\right)(\cdot)$ be the unique solution that satisfies

$$
\frac{d p(t)}{d t} \in-\partial V(p(t)), p(0)=p_{0} .
$$

Then for any series of partitions $\Gamma_{\imath}$ such that $\pi_{\Gamma_{\imath}}$ approaches 0 , the corresponding approximation solutions or Euler polygonal arcs $p_{\Gamma_{\imath}}(t)$ of (8) defined on $[0, b]$ will converge to the unique solution $p\left(0, p_{0}\right)(\cdot)$.

Note that given a selection $f$ in (8) and a partition $\Gamma$, there is an Euler polygonal arc. Given a selection $f$ in (8) and a sequence of partitions $\Gamma_{\imath}$, there are a family of Euler polygonal arcs. We first show that this family of Euler polygonal arcs converges uniformly to the unique solution of the differential inclusion (17). Then, we show that this uniform convergence holds for all selections $f$ in (8).

Proof. Let $\Gamma_{\imath}=\left\{t_{0}, t_{1}, \cdots, t_{\mu_{\imath}}\right\}$ be a sequence of partitions of $[0, b]$ such that $\pi_{\Gamma_{\imath}} \rightarrow 0$, and (necessarily) $\mu_{\imath} \rightarrow \infty$. Let $p_{\Gamma_{\imath}}$ be the corresponding Euler polygonal arcs.

Given a selection $f$ in (8), let $p_{\Gamma_{\imath}}^{f}$ be the corresponding Euler polygonal arcs for $f$. Note that on the interval $\left(t_{j}, t_{j+1}\right)$ of each partition, we have

$$
\left\|\dot{p}_{\Gamma_{\imath}}^{f}(t)\right\|=\left\|f\left(p\left(t_{j}\right)\right)\right\| \leq \gamma\left\|p\left(t_{j}\right)\right\|+c .
$$

Then from the proof of Clarke et al (1988, pp.183\&184), there are uniform bounds $M$ and $k$ such that

$$
p_{\Gamma_{\imath}}^{f}(0)=p_{0},\left\|p_{\Gamma_{\imath}}^{f}-p_{0}\right\|_{\infty} \leq M,\left\|\dot{p}_{\Gamma_{\imath}}^{f}\right\|_{\infty} \leq k .
$$

Applying Arzela-Ascoli Theorem, there is a subsequence $p_{\Gamma_{\alpha}}^{f}(t)$ that uniformly converges. We know that every Euler solution is a trajectory by Corollary 1.2 of Clarke et al $(1988$, pp.186) of 
(17). Therefore, the uniqueness theorem ${ }^{11}$ implies that the Euler solution is actually the unique solution $p\left(0, p_{0}\right)(\cdot)$ of the differential inclusion $(17)$.

Now let's prove that the whole sequence $p_{\Gamma_{2}}^{f}(t)$ uniformly converges to $p\left(0, p_{0}\right)(\cdot)$ by a contradiction. Suppose, on the contrary, that $p_{\Gamma_{\iota}}^{f}(t)$ doesn't uniformly converge, then there exist $\epsilon_{0}>0$ and a subsequence $p_{\Gamma_{\beta}}^{f}(t)$ such that

$$
\left\|p_{\Gamma_{\beta}}^{f}-p\left(0, p_{0}\right)\right\|_{\infty}>\epsilon_{0}
$$

However, the subsequence $p_{\Gamma_{\beta}}^{f}(t)$ also satisfies (18). Therefore the same argument leads to another subsequence of $p_{\Gamma_{\beta}}^{f}(t)$ that uniformly converges to $p\left(0, p_{0}\right)(\cdot)$, which apparently contradicts (19). We reach a contradiction and this proves our claim that the whole sequence $p_{\Gamma_{\imath}}^{f}(t)$ uniformly converges to $p\left(0, p_{0}\right)(\cdot)$. Since this is true for an arbitrary $f$ and the Euler solution is the unique solution of the differential inclusion (17), it follows that all Euler polygonal arcs $p_{\Gamma_{\imath}}^{f}(t)$ converges uniformly to $p\left(0, p_{0}\right)(\cdot)$ for all selections $f$ in $(8)$. This shows that $p_{\Gamma_{\imath}}$ converges uniformly to $p\left(0, p_{0}\right)(\cdot)$.

Given a partition $\Gamma$, define $\pi_{\Gamma}:[0, b] \rightarrow R$ by $\pi_{\Gamma}(t)=\max \left\{t_{j}-t_{j-1}: \forall j\right.$ s.t. $\left.t_{j-1} \geq t\right\}$. A partition $\Gamma_{k}$ is a refinement of the other partition $\Gamma_{k^{\prime}}$, denoted by $\Gamma_{k} \sqsupset \Gamma_{k^{\prime}}$, if $\pi_{\Gamma_{k}}(t) \leq \pi_{\Gamma_{k^{\prime}}}(t)$ for all $t \in[0, b]$ and the inequality is strict for some $t$.

Given $\epsilon>0$, define

$$
W_{\epsilon}=\left\{p \in R_{+}^{n}: d_{W}(p) \leq \epsilon\right\},
$$

where $d_{W}(p)$ is the distance function defined by

$$
d_{W}(p)=\inf \{\|p-q\|: q \in W\} .
$$

The set $W_{\epsilon}$ is the one that is $\epsilon$-close to $W$. An application of Theorems 20 and 16 is as follows:

Corollary 21. For any $\epsilon>0$, there exist a sequence of partitions $\Gamma_{1}, \cdots, \Gamma_{\alpha}, \Gamma_{\alpha+1}, \cdots$ of $[0, b]$ such that $\Gamma_{\alpha} \sqsubset \Gamma_{\alpha+1}, \alpha=1,2, \cdots$, and an $\alpha^{*}$ such that for all $\alpha \geq \alpha^{*}$ and all Euler polygonal arcs $p_{\Gamma_{\alpha}}$ of (8), there exists a finite $T$ such that

$$
p_{\Gamma_{\alpha}}(t) \in W_{\epsilon}, \forall t \geq T \text {. }
$$

Proof. Let $\Gamma_{1}, \cdots, \Gamma_{\alpha}, \Gamma_{\alpha+1}, \cdots$ be a sequence of uniform partitions of $[0, b]$ such that $\Gamma_{\alpha} \sqsubset$ $\Gamma_{\alpha+1}, \alpha=1,2, \cdots$. Theorem 16 shows that $p\left(0, p_{0}\right)(\cdot)$ reaches $W$ in finite time. Theorem 20 shows that there exists a partition $\Gamma_{\alpha^{*}}$ such that for all partitions $\Gamma_{\alpha}$ that are refinements of $\Gamma_{\alpha^{*}}$, all polygonal arcs $p_{\Gamma_{\alpha}}$ of (8) uniformly converge to $p\left(0, p_{0}\right)(\cdot)$. This means that given $\epsilon>0$, a finite time $T$ exists such that $p_{\Gamma_{\alpha}}(t) \in W_{\epsilon}$ for all $t \geq T$.

Suppose that a Walrasian equilibrium exists. Then it follows from Corollary 21 that given $\epsilon>0$, a partition $\Gamma_{\alpha^{*}}$ can be found such that all Euler polygonal arcs $p_{\Gamma_{\alpha}}$ defined by (8) converge

\footnotetext{
${ }^{11}$ This follows from the uniqueness theorem of Yotrutani (1978) and the continuity of $u \mapsto p\left(u, p_{0}\right)$ shown by Hu and Papageorgiou (1998, pp.8). Alternatively, one may directly apply the uniqueness result in Theorem 1 in Aubin and Cellina (1984, pp.159) if $b \rightarrow \infty$ and the Euler polygonal arcs are extended to the interval $[0, \infty)$. We use the result in Yotrutani (1978) here and in the proof of Theorem 15 because we are more interested in the situation where the Euler polygonal arcs are defined on $[0, b]$, where $b$ is a large but finite number.
} 
to the Walrasian equilibrium prices with $\epsilon$-accuracy in finite time for all partitions $\Gamma_{\alpha}$ that are refinements of the partition $\Gamma_{\alpha^{*}}$. Moreover, all Euler polygonal arcs will never come out thereafter once they reach there. Now in an economy that does not have a Walrasian equilibrium, Corollary 21 shows that a partition $\Gamma_{\alpha^{*}}$ can be found such that all Euler polygonal arcs $p_{\Gamma_{\alpha}}$ defined by (8) converge to the market equilibrium prices with $\epsilon$-accuracy in finite time for all partitions $\Gamma_{\alpha}$ that are refinements of the partition $\Gamma_{\alpha^{*}}$. Again, once they have arrived there, they will stay thereafter.

Let $\Gamma$ be a partition of $[0, b]$ with strictly decreasing increments, i.e., the diameter function $t \mapsto \pi_{\Gamma}(t)$ is strictly decreasing. Then, Corollary 21 shows that given any $\epsilon>0$, there always exist such a partition $\Gamma$ and a time $t^{*}$ such that all Euler polygonal arcs $p_{\Gamma}$ defined by (8) stay in the set $W_{\epsilon}$ for all $t \geq t^{*}$. This result is very helpful for the design of an auction to discover the Walrasian or market equilibrium prices. Note that the interval $[0, b]$ is now considered as the increments in the auction not the real time to implement the auction. This is important because the price adjustment process defined in (8) and (9) can be implemented independent of time. Now the continuous time price adjustment process should be understood as a situation where prices are perfectly flexible. The discrete time price adjustment process should be understood as a situation where there are some rigidities in the price system. As the price system becomes more and more flexible, our results show that the Euler price adjustment process defined in (8) converge to the Walrasian equilibrium prices, if any, or otherwise, to the market equilibrium prices, which always exist.

\section{Implementation}

We have shown above that a simple price adjustment process exists that converges to a price vector in finite time that minimizes the economic rent function $V(p)$, no matter whether an economy has a Walrasian equilibrium or not. This can be used in the design of an auction mechanism for the sale of multiple indivisible objects. To do so, the first step is to choose a proper price adjustment increment. One way to do this is to choose an increment sequence of $\frac{\delta}{t}$, where $\delta$ is a fixed number and $t$ is the time to make the adjustment. Such a sequence converges to zero as time $t$ approaches infinity. Our result shows that with such a sequence of increments, all our price adjustment processes will converge to a price vector $p$ in $W$. This accomplishes the step to discover the prices. The second step is to design a game to implement the map $\mathcal{X}^{*}(p)$ of allocations in Nash equilibrium. This is especially important for an economy in which there is no Walrasian equilibrium. Here we present in one way how this can be done.

An agent's preference over object bundles $2^{N}$ is a linear order on the set $2^{N}$ of all subsets of objects $N$. Let $\succ$ denote the set of all such preferences. The seller's preference is a linear order on the set of all feasible allocations. Let $\succ^{0}$ denote the set of all such preferences for the seller.

Given a preference profile $\succeq=\left(\succeq_{0} ; \succeq_{1}, \cdots, \succeq_{m}\right) \in \succ^{0} \times \succ^{1} \times \cdots \succ^{m}$. A feasible allocation $X$ is Pareto optimal with respect to $\succeq$ if for all feasible allocations $Y$, we have that $X \succeq_{0} Y$ and $X_{i} \succeq_{i} Y_{i}$ for all $i \in M$. It is individually rational with respect to $\succeq$ if $X \succeq_{0} \emptyset$, and $X_{i} \succeq_{i} \emptyset$ for all $i \in M$, where $\emptyset$ denotes the allocation in which each agent buyer is allocated no objects in the seller's case or the empty set in the agent buyers' case. Let $P I(\succeq)$ denote the set of all Pareto optimal (PO) and individually rational (IR) allocations with respect to $\succeq$.

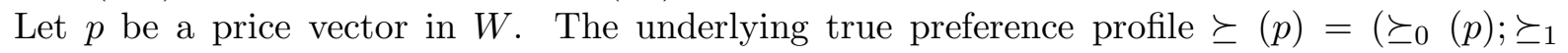
$\left.(p), \cdots, \succeq_{m}(p)\right)$ is defined as follows. For the seller $0, X \succeq_{0}(p) Y$ iff $\sum_{i \in M} \sum_{a \in X_{i}} p_{a} \geq \sum_{i \in M} \sum_{a \in Y_{i}} p_{a}$ for all feasible allocations $X, Y$. For each buyer $i \in M, S \succeq_{i}(p) T$ iff $v_{i}(S, p) \geq v_{i}(T, p)$ for all object bundles $S, T \in 2^{N}$.

Now consider the implementation game $\Gamma=\left(\succ^{0} \times \succ^{1} \times \cdots \times \succ^{m}, \varphi ; \succeq(p)\right)$. The set $\succ^{0} \times \succ^{1}$ 
$\times \cdots \times \succ^{m}$ is the set of reporting strategies for the seller and agent buyers respectively. $\varphi$ is an outcome function passing from $\succ^{0} \times \succ^{1} \times \cdots \times \succ^{m}$ into the set of all feasible allocations. The profile $\succeq(p)$ is the underlying true preference profile. Without confusion, we will use $\succeq$ instead $\succeq(p)$ for the underlying true preferences. We are interested in the situation where the outcome function $\varphi$

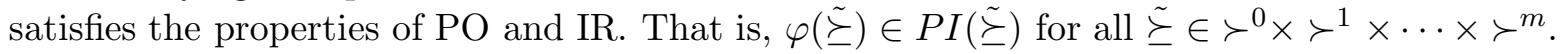

Note that for a fixed price vector $p \in W$, a feasible allocation of objects is equivalent to a matching between objects and agent buyers in the two-sided matching markets (Roth and Sotomayor (1990)). We follow Roth and Vande Vate (1991) and Roth and Rothblum (1999) to consider a class of simple strategies called "truncations" in the two-sided matching markets. A truncation strategy for an agent buyer is a preference ordering which is order-consistent with his or her true preference but has fewer acceptable object bundles ${ }^{12}$. A truncation strategy for the seller is a preference ordering which is order-consistent with his or her true preference but has fewer acceptable allocations ${ }^{13}$. This class of strategies excludes some complicated strategies like the changes in orders that may be profitable. But when players have very little information about the other players' preferences, Roth and Rothblum (1999) convincingly showed why it is plausible for players to consider this class of profitable strategies in truncations in the job matching markets. In our situation, we have the following result.

Claim 22. Given any strategy profile $\left(\Xi_{-j}, \Xi_{j}\right)$ that is not necessarily truncated, there always exists a truncated strategy $\check{\succeq}_{j}$ for an agent $j$ such that

$$
\varphi_{j}\left(\left(\succeq_{-j}, \check{\succeq}_{j}\right)\right) \succeq_{j} \varphi_{j}\left(\left(\succeq_{-j}, \succeq_{j}\right)\right)
$$

Proof. If $\varphi_{j}\left(\left(\succeq_{-j}, \succeq_{j}\right)\right)$ is not an empty bundle, then let $\check{\succeq}_{j}$ be a strategy for $j$ truncated up to the bundle $\varphi_{j}\left(\left(\succeq_{-j}, \succeq_{j}\right)\right)$ which is then immediately followed by the empty bundle ${ }^{14}$. If $\varphi_{j}\left(\left(\Xi_{-j}, \Xi_{j}\right)\right)$ is empty, then let $\check{\succeq}_{j}$ be the true preference of agent $j$. Note that the allocation $\varphi\left(\left(\check{\succeq}_{-j}, \check{\succeq}_{j}\right)\right)$ is feasible and satisfies IR with respect to the profile $\left(\check{\succeq}_{-j}, \check{\Xi}_{j}\right)$. Since $\varphi$ is IR and $\mathrm{PO}$, it follows that

$$
\varphi_{j}\left(\left(\succeq_{-j}, \check{\succeq}_{j}\right)\right) \succeq_{j} \varphi_{j}\left(\left(\succeq_{-j}, \succeq_{j}\right)\right) .
$$

This completes the proof.

Claim 22 is also true if it is the seller. The proof is quite similar and thus omitted. Given a profile $\succeq$ of strategies, the seller or the agent buyers can simply focus on reporting truncated strategies since the class of truncated strategies can service the players at least as good as some other more complicated strategies like the changes in orders. A Nash Equilibrium in truncation is a Nash equilibrium when strategies are all in truncations.

Theorem 23. Let $p$ be a price vector in $W$ and $\varphi$ be a $P O$ and IR outcome function in the game $\Gamma$. For every Nash equilibrium $\succeq^{*}$ in truncations, $\varphi\left(\succeq^{*}\right) \in \mathcal{X}^{*}(p)$. Moreover, for every allocation $X \in \mathcal{X}^{*}(p)$, there is a Nash equilibrium $\succeq^{*}$ in truncations such that $\varphi\left(\succeq^{*}\right)=X$.

\footnotetext{
${ }^{12}$ An object bundle is acceptable for an agent if he/she prefers it to the empty bundle.

${ }^{13} \mathrm{An}$ allocation is acceptable for the seller if he/she prefers it to the allocation in which each agent is allocated the empty bundle.

${ }^{14}$ The order after the empty bundle does not matter for us.
} 
Proof. Note that $\mathcal{X}^{*}(p)$ is the set of all PO and IR allocations with respect to the underlying true preference profile $\succeq$. Note also that $\varphi\left(\succeq^{*}\right)$ is IR, since $\succeq^{*}$ is a Nash equilibrium. Now suppose that $\varphi\left(\succeq^{*}\right)$ is not in $\mathcal{X}^{*}(p)$. Then this means that there exists a feasible allocation $X$ such that the seller and all agent buyers weakly prefer $X$ to $\varphi\left(\succeq^{*}\right)$, and at least one of them strictly prefers $X$ to $\varphi\left(\succeq^{*}\right)$, with respect to the underlying true profile $\succeq$. If $\varphi_{i}\left(\succeq^{*}\right)$ is nonempty for all $i \in M$, the fact that $\succeq^{*}$ is a truncation profile implies that $\varphi\left(\succeq^{*}\right)$ is not PO with respect to $\succeq^{*}$.

Let $\mathcal{T}$ denote the set of agents $i$ such that $\varphi_{i}\left(\succeq^{*}\right)$ is empty and $X_{i}$ is not. If $\mathcal{T}$ is empty, then it follows again from the fact that $\succeq^{*}$ is a truncation that $\varphi\left(\succeq^{*}\right)$ is not PO for $\succeq^{*}$. So suppose that $\mathcal{T}$ is not empty. Pick any $i$ from $\mathcal{T}$. Consider a strategy $\succeq_{i}^{\prime}$ that is a truncation up to the bundle $X_{i}$ which is then immediately followed by the empty set. Note that since $X$ is a feasible allocation and IR with respect to $\succeq^{*}$, it is a feasible allocation and IR with respect to the profile $\left(\succeq_{-i}^{*}, \succeq_{i}^{\prime}\right)$. Since $\varphi$ is IR and PO, it follows that $\varphi_{i}\left(\left(\succeq_{-i}^{*}, \succeq_{i}^{\prime}\right)\right) \succeq_{i} X_{i} \succ_{i} \emptyset=\varphi_{i}\left(\succeq^{*}\right)$. But this shows that $\succeq^{*}$ is not a Nash equilibrium. This completes the proof that $\varphi\left(\succeq^{*}\right) \in \mathcal{X}^{*}(p)$.

To show the second part, let $X$ be a feasible allocation in $\mathcal{X}^{*}(p)$. For an agent $i \in M$, if $X_{i}$ is nonempty, then let $\check{\succeq}_{i}$ be a strategy that is a truncation up to the bundle $X_{i}{ }^{15}$ If $X_{i}$ is empty, then let $\tilde{\succeq}_{i}$ be the true preference. For the seller, let $\check{\succeq}_{0}$ be the truncation up to the allocation $X$. We show that the profile $\check{\succeq}=\left(\check{\succeq}_{0}, \tilde{\succeq}_{1}, \cdots, \tilde{\succeq}_{m}\right)$ is a Nash equilibrium of the game $\Gamma$. Note that by

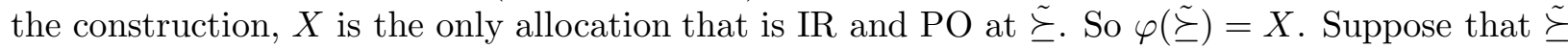
is not a Nash equilibrium. Then there exists a player $j$ such that $\varphi_{j}\left(\check{\succeq}_{-j}, \succeq_{j}^{\prime \prime}\right) \succ_{j} \varphi_{j}(\tilde{\succeq})$. Since $\check{\succeq}$ is a truncation and $\varphi\left(\left(\check{\succeq}_{-j}, \succeq_{j}^{\prime \prime}\right)\right)$ is feasible and PO at $\left(\check{\succeq}_{-j}, \succeq_{j}^{\prime \prime}\right)$, this implies that $\varphi$ is not PO at

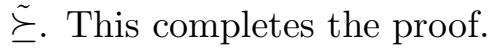

Therefore, Theorem 23 shows that the map $\mathcal{X}^{*}(p)$ that satisfies the Edgeworth trading hypothesis can be implemented by a strategic game induced by an IR and PO allocation function in Nash equilibrium with truncations.

\footnotetext{
${ }^{15}$ Also if there is any bundle $S$ such that agent is indifferent between $S$ and $X_{i}$, put the empty bundle in front of $S$ in the construction of $\check{\succeq}_{i}$.
} 


\section{References}

[1] J. Aubin and A. Cellina, Differential Inclusions-Set-Valued Maps and Viability Theory, Springer-Verlag, 1984.

[2] S. Bikhchandani and J.W. Mamer, Competitive equilibrium in an exchange economy with indivisibilities, J. Econ. Theory 74 (1997), 385-413.

[3] F. Clarke, Y. Ledyaev, R. Stern, and P. Wolenski, Nonsmooth Analysis and Control Theory, Springer-Verlag, 1988.

[4] V. Danilov and G. Koshevoy, Discrete Convexity and Equilibria, 2001, forthcoming.

[5] G. Demange, D. Gale, and M. Sotomayor, Multi-Item Auctions, Journal of Political Economy 94, 863-872.

[6] F. Fisher, Disequilibrium Foundations of Equilibrium Economics, Cambridge University Press, 1983.

[7] D. Gale and L.S. Shapley: College admissions and the stability of marriage, American Mathematical Monthly 69 (1962), 9-15.

[8] R. Garratt, Decentralizing Lottery Allocations in Markets with Indivisible Commodities, Economic Theory 5, 295-313.

[9] R. Garratt and C.Z. Qin, On a Market for Coalitions with Indivisible Agents and Lotteries, Journal of Economic Theory 77, 81-101.

[10] R. Garratt, T. Keister, C.Z. Qin and K. Shell, Equilibrium Prices When the Sunspot Variable Is Continuous, Journal of Economic Theory 96 (2001), 70-96.

[11] R. Garratt, T. Keister and K. Shell, Comparing Sunspot Equilibrium and Lottery Equilibrium Allocations: The Finite Case, mimeo, 2001.

[12] F. Gül and E. Stacchetti, Walrasian equilibrium with Gross Subsitutes, Journal of Economic Theory 87 (1999), 95-124.

[13] F. Gül and E. Stacchetti, The English Auction with Differentiated Commodities, Journal of Economic Theory 92, 2000, 66-95.

[14] S. Hu and N. Papageorgiou, Time-Dependent Subdifferential Evolution Inclusions and Optimal Control, Memoirs of the American Mathematical Society, Number 632, American Mathematical Society, 1998.

[15] A. S. Kelso, Jr. and V.P. Crawford, Job matching, coalition formation, and gross substitutes, Econometrica 50 (1982), 1483-1504.

[16] G. Laan, D. Talman, and Z. Yang, Existence and Welfare Properties of Equilibrium on an Exchange Economy with Multiple Divisible and Indivisible Commodities and Linear Technologies, Journal of Economic Theory 96 (2001), 1-18.

[17] J. Ma, Competitive equilibrium with indivisibilities, J. Econ. Theory 82(1998a), 458-468. 
[18] J. Ma, English auctions and Walrasian equilibria with multiple objects, Mimeo, 1998(b), Rutgers University-Camden.

[19] P. Milgrom, Put Auction Theory to Work, Journal of Political Economy 108 (2000), 245-272.

[20] P. Milgrom and I. Segal, Envelope Theorems for Arbitrary Choice Sets, Mimeo, 2000, Stanford University, Forthcoming in Econometrica.

[21] E. Prescott and R. Townsend, Pareto Optima and Competitive Equilibria with Adverse Selection and Moral Hazard, Econometrica 52 (1984), 21-45.

[22] Roth, A.E., and Rothblum, U. (1999), "Truncation Strategies in Matching Markets-in search of advice for participants," Econometrica 67, 21-44.

[23] Roth, A.E., Sotomayor, M.: Two-sided Matching: a Study in Game-theoretic Modeling and Analysis, Cambridge University Press, Cambridge 1990.

[24] Roth, A.E., Vande Vate, J.H. (1991): "Incentives in Two-sided Matching with Random Stable Mechanisms," Economic Theory, 1, 31-44.

[25] H. Royden, Real Analysis, 3rd Edition, Macmillan Publishing Company, 1988.

[26] Shapley, Lloyd, and Martin Shubik (1972): "The Assignment Game I: the core," International Journal of Game Theory 1, 111-30.

[27] V. Smith, An experimental study of competitive market behavior," Journal of Political Economy 70 (1962), 111-137.

[28] V. Smith, Experimental auction markets and the Walrasian hypothesis," Journal of Political Economy 73 (1965), 387-393 